\title{
Magnetic Moments of Short-Lived Nuclei with Part-per-Million Accuracy: Toward Novel Applications of $\beta$-Detected NMR in Physics, Chemistry, and Biology
}

R. D. Harding $\odot,{ }^{1,2, \dagger}$ S. Pallada $\odot,{ }^{1, \$}$ J. Croese $\odot,{ }^{1,3}$ A. Antušek $\odot,{ }^{4}$ M. Baranowski $\odot,{ }^{5}$ M. L. Bissellø, ${ }^{6}$ L. Cerato $\odot,{ }^{7}$ K. M. Dziubinska-Kühn $\odot,{ }^{1,8}$ W. Gins $\odot,{ }^{9,8}$ F. P. Gustafsson $\odot,{ }^{9}$ A. Javaji, ${ }^{1,10,}$ R. B. Jolivet $\odot,{ }^{3}$ A. Kanellakopoulos $\odot,{ }^{9}$ B. Karg $\odot,{ }^{3}$ M. Kempka $\odot,{ }^{5}$ V. Kocman $\odot,{ }^{11}$ M. Kozak $\odot,{ }^{5,12}$ K. Kulesz $\odot,{ }^{3,1}$ M. Madurga Flores $\odot,{ }^{13}$ G. Neyens $\odot,{ }^{9,1}$ R. Pietrzyk ${ }^{5}$ J. Plavec $\odot,{ }^{11}$ M. Pomorski, ${ }^{14, * *}$ A. Skrzypczak $\odot,{ }^{15}$ P. Wagenknecht,${ }^{1,10,+\dagger}$ F. Wienholtz $\odot,{ }^{1, *}$ J. Wolak, Z. Xu® ${ }^{13}$ D. Zakoucky, ${ }^{16}$ and M. Kowalska $\odot^{1,3, *}$

${ }^{1}$ Experimental Physics Department, CERN, 1211 Geneva, Switzerland

${ }^{2}$ Department of Physics, University of York, YO10 5DD York, United Kingdom

${ }^{3}$ Department of Nuclear and Particle Physics, University of Geneva, 1211 Geneva, Switzerland

${ }^{4}$ Faculty of Materials Science and Technology, Slovak University of Technology, 91724 Trnava, Slovak Republic

${ }^{5}$ Faculty of Physics, Adam Mickiewicz University, 61-614 Poznań, Poland

${ }^{6}$ School of Physics and Astronomy, The University of Manchester, M13 9PL Manchester, United Kingdom

${ }^{7}$ Department of Molecular Biology, University of Geneva, 1211 Geneva, Switzerland

${ }^{8}$ Institute of Analytical Chemistry, Leipzig University, D-04103 Leipzig, Germany

${ }^{9}$ Instituut voor Kern- en Stalingsfysica, KU Leuven, B-3001 Leuven, Belgium

${ }^{10}$ Faculty of Mathematics and Natural Sciences, Oldenburg University, 26129 Oldenburg, Germany

${ }^{11}$ Slovenian NMR Centre, National Institute of Chemistry, SI-1001 Ljubljana, Slovenia

${ }^{12}$ National Synchrotron Radiation Centre SOLARIS, Jagiellonian University, 30-392 Kraków, Poland

${ }^{13}$ Department of Physics and Astronomy, University of Tennessee, Knoxville 37996, Tennessee, USA

${ }^{14}$ Faculty of Physics, University of Warsaw, 02-093 Warsaw, Poland

${ }^{15}$ Faculty of Chemical Technology, Poznan University of Technology, 60-965 Poznan, Poland

${ }^{16}$ Nuclear Physics Institute, Czech Academy of Sciences, CZ-25068 Rez, Czech Republic

(Received 25 February 2020; revised 4 September 2020; accepted 20 November 2020; published 28 December 2020)

We determine for the first time the magnetic dipole moment of a short-lived nucleus with part-permillion (ppm) accuracy. To achieve this 2-orders-of-magnitude improvement over previous studies, we implement a number of innovations into our $\beta$-detected nuclear magnetic resonance $(\beta$-NMR) setup at ISOLDE at CERN. Using liquid samples as hosts, we obtain narrow, subkilohertz-linewidth, resonances, while a simultaneous in situ ${ }^{1} \mathrm{H}$ NMR measurement allows us to calibrate and stabilize the magnetic field to ppm precision, thus eliminating the need for additional $\beta$-NMR reference measurements. Furthermore, we use $a b$ initio calculations of NMR shielding constants to improve the accuracy of the reference magnetic moment, thus removing a large systematic error. We demonstrate the potential of this combined approach with the $1.1 \mathrm{~s}$ half-life radioactive nucleus ${ }^{26} \mathrm{Na}$, which is relevant for biochemical studies. Our technique can be readily extended to other isotopic chains, providing accurate magnetic moments for many short-lived nuclei. Furthermore, we discuss how our approach can

\footnotetext{
* Corresponding author.

kowalska@cern.ch

†Present address: Department of Medical Physics and Clinical Engineering, Nottingham University Hospitals NHS Trust, Nottingham NG7 2UH, United Kingdom.

"Present address: HEPIA Geneva, HES-SO, 1202 Geneva, Switzerland.

${ }^{\S}$ Present address: Department of Physics, University of Jyväskylä, 40014 Jyväskylä, Finland.

"Present address: Department of Physics and Astronomy, University of British Columbia, V6T 1Z4 Vancouver, Canada.

*** Present address: University of Bordeaux, CNRS, CENBG, F-33170 Gradignan, France.

${ }^{\dagger}$ Present address: University of Tennessee, Knoxville 37996, Tennessee, USA.

Present address: Nuclear Physics Institute, Technical University Darmstadt, 64289 Darmstadt, Germany.
}

Published by the American Physical Society under the terms of the Creative Commons Attribution 4.0 International license. Further distribution of this work must maintain attribution to the author(s) and the published article's title, journal citation, and DOI. 
open the path toward a wide range of applications of the ultrasensitive $\beta$-NMR in physics, chemistry, and biology.

DOI: 10.1103/PhysRevX.10.041061

Subject Areas: Chemical Physics, Interdisciplinary Physics, Nuclear Physics

\section{INTRODUCTION}

The magnetic dipole moment $\mu$ is a fundamental property of atomic nuclei, and it is one of the primary observables used to investigate the nuclear wave function [1-10]. At the same time, it serves as a versatile probe to measure the local magnetic field at the nucleus. This ability lies at the core of various spectroscopic techniques, among which a prominent role is played by nuclear magnetic resonance (NMR), which is an indispensable tool for determining structural details and dynamics in chemistry, biology, and materials science [11,12].

In NMR experiments, one measures the Larmor frequency $\nu_{L}$ of nuclei of spin $I$ precessing in a magnetic field. This frequency is the product of the gyromagnetic ratio $\gamma$ of the nucleus and the local magnetic field $B$ at the site of the nucleus, i.e., the applied magnetic field corrected for the effect of the electrons in the sample:

$$
\nu_{L}=\frac{\gamma B}{2 \pi}=\frac{\mu B}{h I} .
$$

If one wants to employ NMR to extract a nuclear magnetic moment $\mu$, two inputs are, thus, essential. First, the Larmor frequency $\nu_{L}$ must be measured. For stable nuclei, $\nu_{L}$ has been determined with sub-part-per-million precision since the early years of NMR [13-16] and, thus, is not the dominant source of uncertainty in the derived magnetic moment. The second essential input to derive $\mu$ is the NMR shielding, describing the local effect of electrons in the sample on the applied magnetic field. Until recently, this effect has been poorly quantified and sometimes even neglected [17]. However, the introduction of reliable NMR shielding constants, provided by modern $a b$ initio methods [18,19], enabled correction of this source of the systematic error in nuclear magnetic moment data [20,21], which in extreme cases reached per-mill or percent levels [22-25]. This correction turned out to be crucial for the tests of QED in the strong electromagnetic fields of highly charged ions [26,27]. Here, a more accurate value of the magnetic moment of ${ }^{209} \mathrm{Bi}[23,24]$ resolved a significant discrepancy between the measured and predicted hyperfine splitting (an effect of the interaction between $\mu$ and the magnetic field produced by the atomic electrons) of highly charged ${ }^{209} \mathrm{Bi}$ [28], showing that QED is still valid in such a strong magnetic field. Based on the corrected magnetic moments, a new referencing scheme in NMR spectroscopy was also proposed [29], which allows a direct measurement of the NMR shielding instead of a chemical shift (i.e., a difference in NMR shieldings in different hosts).
More accurate nuclear magnetic moments can clearly bring new applications in different fields of research, as shown above for stable nuclei. However, magnetic moments of short-lived nuclei have not yet been measured with equally small uncertainty. In the present work, we demonstrate for the first time the determination of a magnetic moment of a short-lived nucleus with part-permillion (ppm) accuracy. This achievement is shown on the $1.1 \mathrm{~s}$ half-life ${ }^{26} \mathrm{Na}$ using an improved version of the $\beta$-NMR technique, combined with ab initio calculations of NMR shielding for the stable reference ${ }^{23} \mathrm{Na}$. The isotope ${ }^{26} \mathrm{Na}$ is used for the proof-of-principle experiment because of sodium's importance for biochemistry applications [30].

The $\beta$-NMR technique is based on the directional asymmetry of $\beta$-particle emission from spin-polarized $\beta$-decaying nuclei $[31,32]$. The most attractive feature of the method is its sensitivity, which is up to $10^{10}$ times higher than in conventional NMR [33], with down to $10^{6}$ resonating nuclei leading to an NMR spectrum. The technique has been applied to measure the magnetic moments of short-lived nuclei down to per-mill precision [3,34-43] and for electronic, magnetic, and structural investigations in materials science [44-51]. However, in chemistry and biology, $\beta$ NMR is far from being a routinely applicable spectroscopic method $[33,52,53]$, due to numerous experimental challenges. One of them is the requirement of time-consuming reference measurements with the same short-lived nucleus in a different chemical environment [52,54]. Furthermore, those reference measurements are performed in a solid-state sample [52,53], resulting in relatively wide resonance signals, thus increasing the final error on the extracted experimental value. Another challenge is due to the reduced precision and accuracy in the measured frequency and deduced magnetic moments, which prevent a direct comparison of the data with results from conventional NMR and from ab initio chemical calculations of local fields. The work presented here addresses all of the above limitations of the $\beta$-NMR technique, with the key ingredient being an accurate magnetic moment measurement.

The developments presented here are crucial for future applications of high-precision $\beta$-NMR spectroscopy using a variety of radioactive probes, not only in the fields of chemistry and biology, but also for nuclear structure research. For example, determining the neutron distribution in light neutron-rich nuclei $[55,56]$ is experimentally very challenging, as neutrons do not carry electric charge. However, one can access this information by measuring the distribution of magnetization inside exotic nuclei. This approach requires very high-precision magnetic moment 


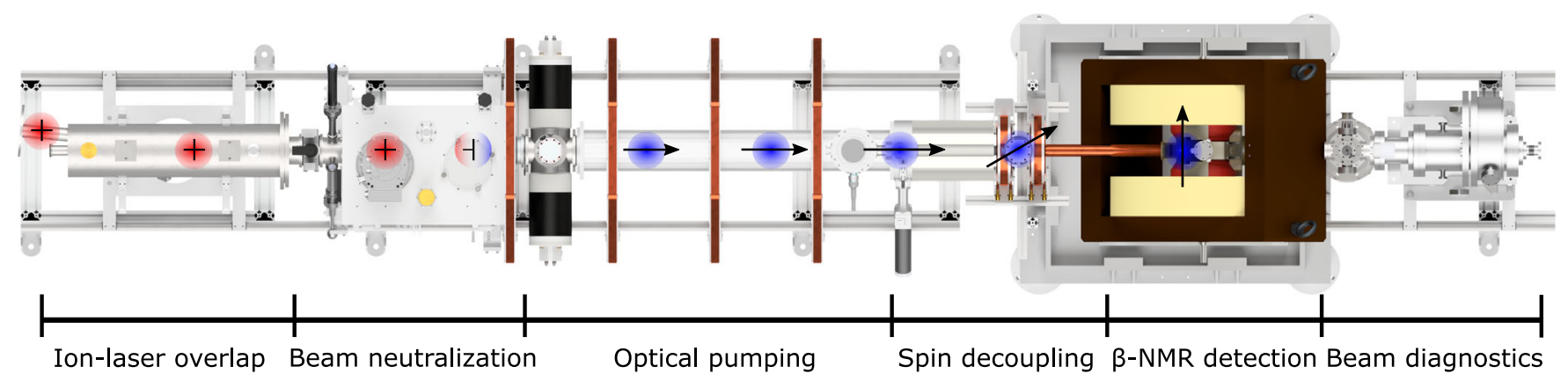

FIG. 1. Top view of the laser polarization and $\beta$-NMR beam line $[61,62]$. The ion and laser beams enter from the left. The ions are represented by red circles with a plus sign. The neutral atoms are represented by blue circles. The polarization of the atom is represented by arrows. See the text for further details.

measurements, combined with high-precision hyperfinestructure measurements on the same isotope, to be sensitive to the "hyperfine anomaly" [57-59].

\section{TECHNIQUES}

\section{A. $\beta$-NMR on short-lived ${ }^{26} \mathrm{Na}$}

$\beta$-NMR studies are performed on laser-polarized shortlived ${ }^{26} \mathrm{Na}$. The nuclei are produced at the ISOLDE facility at CERN [60], in reactions induced by a $1.4-\mathrm{GeV}$ proton beam of up to $2 \mu \mathrm{A}$, impinging every $3.6 \mathrm{~s}$ on a $\mathrm{UC}_{x}$ (uranium carbide) target. After fast diffusion out of the heated target, sodium atoms are surface ionized, accelerated to $50 \mathrm{keV}$, and mass separated using the ISOLDE high resolution separator HRS. The pure isotopic beam of ${ }^{26} \mathrm{Na}$, with an intensity of $2-5 \times 10^{7}$ ions per second, is transported to the laser polarization beam line [61,62] shown in Fig. 1.

There, the ${ }^{26} \mathrm{Na}^{+}$beam is overlapped with circularly polarized laser light. Next, it passes through a neutralization cell, where it picks up an electron as it travels through a vapor of stable ${ }^{23} \mathrm{Na}$. Over the next $1.5 \mathrm{~m}$, the neutral atomic ${ }^{26} \mathrm{Na}$ beam is polarized via optical pumping in the D2 line at $589 \mathrm{~nm}$ [61]. This polarization takes place in a weak guiding magnetic field of $2 \mathrm{mT}$ (applied along the beam path), which defines the quantization axis and prevents the coupling of the electron spins to possible stray fields in the surrounding environment. Next, the atoms pass through a transitional field region of approximately 10-20 $\mathrm{mT}$, where the atomic spins undergo an adiabatic rotation toward the perpendicular magnetic field of the NMR magnet. The spin-polarized atoms pass through a collimator and reach a liquid sample located in a vacuum chamber that is placed between the poles of a Bruker BE25 electromagnet set to a field of 1.2 T (Fig. 2). At this point, the nuclear and electronic spins are decoupled, and the nuclear spin couples to the large static field.

The liquid sample is deposited on a sample holder made of mica. The collimated atom beam and the holder have a diameter of $8 \mathrm{~mm}$. Four such sample holders are attached to a sample ladder that can be moved in and out of the beam path. The emitted $\beta$ particles are registered in two pairs of thin organic scintillators, coupled to compact silicon photodetectors. The sample at the center of the electromagnet is surrounded by a 30-mm diameter coil to which an rf signal can be applied. See Fig. 2 for details.
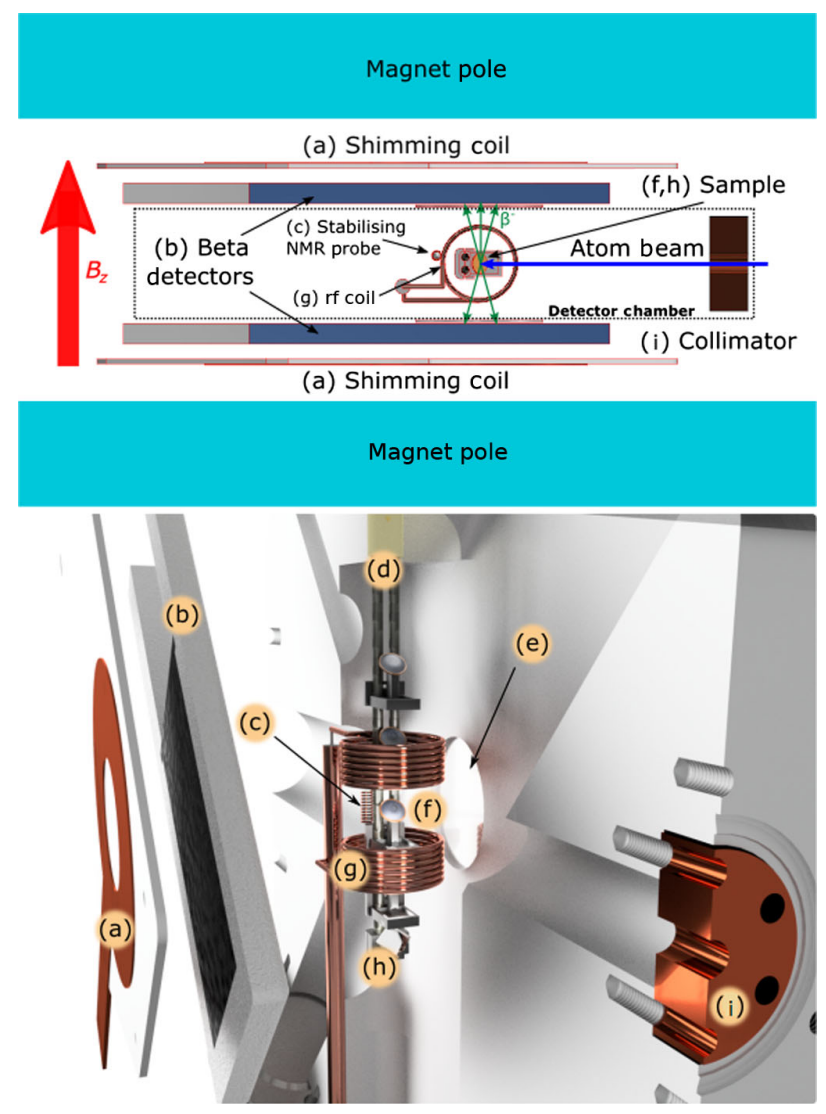

FIG. 2. $\quad \beta$-NMR detection chamber. Top: Schematic cross section as viewed from the top. Bottom: 3D CAD exploded view. (a) Shimming coil to improve field homogeneity; (b) $\beta$-particle detector (plastic scintillator) and Si photomultiplier; (c) ${ }^{1} \mathrm{H}-\mathrm{NMR}$ probe to monitor and actively stabilize the magnetic field; (d) sample ladder; (e) $\beta$-particle window (100 $\mu \mathrm{m}$ aluminum); (f) mica sample holder; (g) main rf coil for NMR excitations;

(h) $\mathrm{NaF}$ crystal to optimize the degree of laser spin polarization;

(i) $8-\mathrm{mm}$ beam collimator. See the text for further details. 


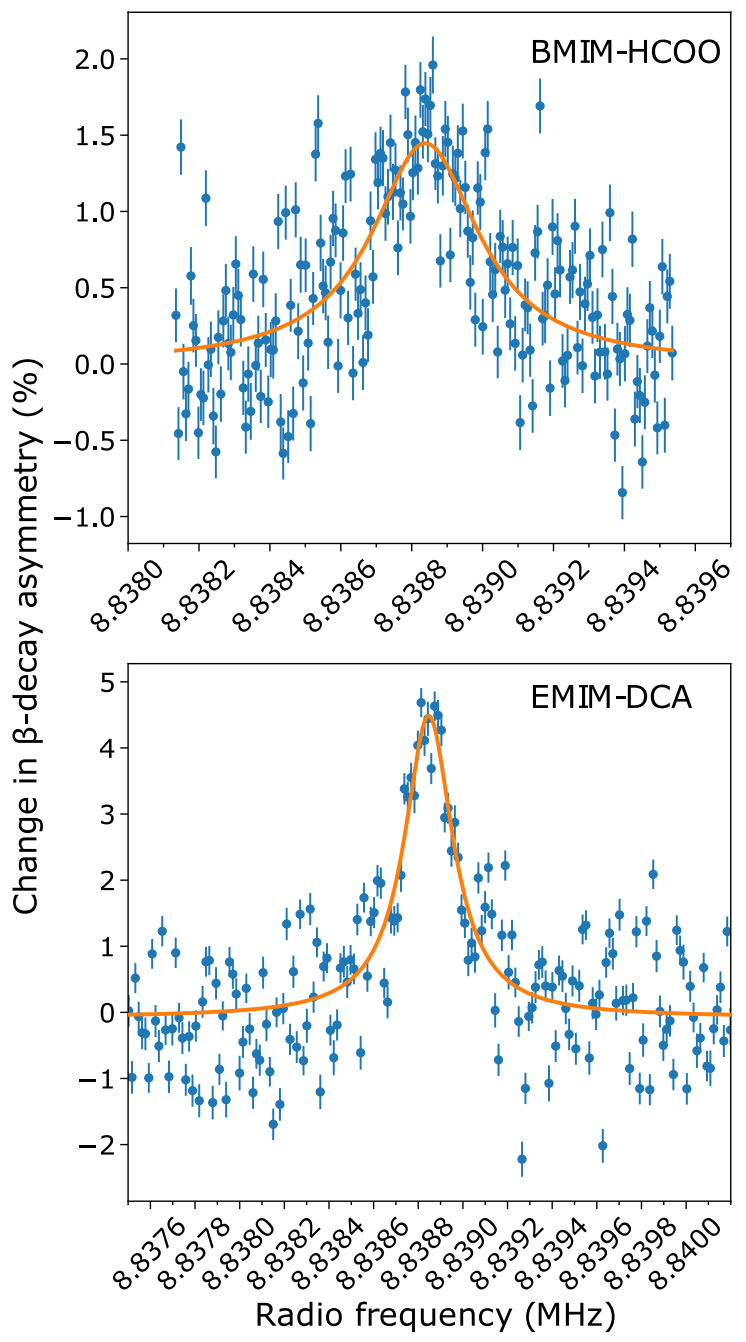

FIG. 3. Examples of ${ }^{26} \mathrm{Na} \beta$-NMR spectra in BMIM-HCOO (top) and EMIM-DCA (bottom). See the text for the description of the fitting procedure. Note the different ranges of $x$ and $y$ axes. The fitted baseline (i.e., experimental asymmetry outside resonance) is subtracted for all data points for an easier comparison of the amplitudes of both signals. The magnetic field is locked to the same ${ }^{1} \mathrm{H}$ frequency for both samples.

To record an NMR spectrum, such as the ones shown in Fig. 3, 200 equally spaced rf frequencies are sequentially set. For each frequency, the ${ }^{26} \mathrm{Na}$ beam is implanted over $200 \mathrm{~ms}$ following the proton-bunch impact. After the start of implantation, the $\beta$ particles are counted for up to $1 \mathrm{~s}$ in the detectors at $0^{\circ}$ and $180^{\circ}$ to the direction of the magnetic field (left and right to the beam axis). From these counts, the experimental $\beta$-decay asymmetry is determined, as a normalized difference in the counts: $\left(N_{0^{\circ}}-N_{180^{\circ}}\right) /\left(N_{0^{\circ}}+N_{180^{\circ}}\right)$. At the same time, the sample is irradiated with a continuous wave rf field of $0.03 \mathrm{mT}$ and a frequency corresponding to the point in the scan. This procedure is repeated for consecutive proton bunches (arriving every 3.6 or $4.8 \mathrm{~s}$ ), to allow most of the nuclei from the previous bunch to decay. If required by the signal-to-noise ratio, several spectra of the same sample can be recorded and summed.

To increase the precision of the NMR measurements to the ppm level, the magnetic field across the sample has to be homogeneous with a temporal stability at the ppm level during a measurement. To ensure the former, a weak magnetic field on the order of $0.02 \mathrm{mT}$ is produced by two shimming coils placed in contact with the magnet poles [63]. In this way, the field homogeneity across the sample volume is improved by more than an order of magnitude in all three axes: $1 \mathrm{ppm}$ along the symmetry axis of the magnet, $3 \mathrm{ppm}$ in the vertical axis, and $5 \mathrm{ppm}$ in the horizontal axis (ion-beam propagation). Since the magnetic field is symmetric with respect to the center of the sample, the remaining inhomogeneity contributes to a broadening of the resonance peak, without a significant shift in the resonance frequency, compared to a pointlike sample. The temporal drift in the magnetic field is addressed using an active stabilization system based on the ${ }^{1} \mathrm{H}$ resonance frequency measured in a tailor-made vacuum-compatible $\mathrm{H}_{2} \mathrm{O}$ NMR probe. The 3-mm diameter probe is located just outside the main excitation rf coil, as shown in Fig. 2, with its middle only $25 \mathrm{~mm}$ away from the center of the sample. The resulting temporal stability is better than $1 \mathrm{ppm}$ between subsecond and 24-h timescales, compared to drifts as big as $1 \mathrm{ppm} /$ minute without it.

Previous $\beta$-NMR studies of the magnetic moments of short-lived nuclei relied on solid-state hosts. For sodium, the studies were performed using a cubic $\mathrm{NaF}$ crystal which retained polarization for several dozen seconds, leading to NMR resonances with the width on the order of $10^{-3}$ of the resonance frequency [42]. In comparison, with liquid-state hosts, it is possible to obtain resonances with over 2 orders of magnitude smaller width (due to molecular tumbling within a liquid [64]), while retaining the nuclear polarization long enough to employ $\beta$-NMR. Unfortunately, most liquid-state hosts used for NMR studies have a high vapor pressure, so when placed inside vacuum they either freeze or evaporate. However, room-temperature ionic liquids, which are salts in a liquid state at room temperature, have an extremely low vapor pressure [65], which makes them suitable hosts for high-precision NMR studies in vacuum environments, as encountered in most $\beta$-NMR setups. For measuring the Larmor frequency of ${ }^{26} \mathrm{Na}$, two different ionic liquids are selected: 1-ethyl-3-methylimidazolium dicyanamide (EMIM-DCA) and 1-butyl-3-methylimidazolium formate (BMIM-HCOO). The EMIM-DCA sample contains approximately $1 \mu \mathrm{M}$ of ${ }^{23} \mathrm{Na}^{+}$, while the BMIMHCOO sample contains $0.5 \mathrm{M}$. Both samples are degassed slowly at $10^{-5}$ mbar pressure for several hours in a separate vacuum chamber. $20 \mu \mathrm{L}$ of each solution is deposited as a 0.4-mm layer on one of the sample holders attached to the sample ladder. The ladder is then placed in the $\beta$-NMR chamber, as shown in Fig. 2, and the pressure inside is lowered slowly from atmospheric pressure to $10^{-5}$ mbar. 
The sample is oriented at $45^{\circ}$ to the atom beam. Because of the high viscosity of both liquids, the layer remains on each substrate at high vacuum for up to $24 \mathrm{~h}$.

\section{B. Conventional NMR on stable ${ }^{23} \mathrm{Na}$}

At the time of investigation, it was not possible to obtain a conventional NMR signal from ${ }^{23} \mathrm{Na}$ at the $\beta$-NMR beam line. Therefore, ${ }^{23} \mathrm{Na}$ and ${ }^{1} \mathrm{H}$ NMR spectra are recorded on a conventional NMR spectrometer. Our earlier systematic NMR studies show that changing ${ }^{23} \mathrm{Na}$ concentration from micromolar to molar ranges and degassing for an extended period shifts the ${ }^{23} \mathrm{Na}$ resonance by less than $0.5 \mathrm{ppm}$. This value is taken as our experimental uncertainty for ${ }^{23} \mathrm{Na}$, and degassing is not carried out during the measurements presented here. The field of $7.05 \mathrm{~T}$ is provided by a Bruker Avance DMX $300 \mathrm{MHz}$ spectrometer, and a basic pulsed-NMR scheme is applied (single $\pi / 2$ rf pulse) on samples kept at room temperature. The sample preparation and $\mathrm{Na}^{+}$concentration are as close as possible to those in the $\beta$-NMR experiment: approximately $1 \mu \mathrm{M}$ in the EMIMDCA sample and $0.5 \mathrm{M}$ in the BMIM-HCOO sample.

For the measurements, $200 \mu \mathrm{L}$ of each solution are sealed inside a 3-mm diameter NMR tube. The tube is placed inside a 5-mm diameter tube filled with $\mathrm{D}_{2} \mathrm{O}$, whose ${ }^{2} \mathrm{H}$ NMR signal is used to stabilize the magnetic field automatically during the measurements (field locking). ${ }^{1} \mathrm{H}$ NMR resonances are also recorded within several minutes from ${ }^{23} \mathrm{Na}$ spectra, using the same setup with two concentric tubes. Here, the $3-\mathrm{mm}$ tube is filled with $\mathrm{H}_{2} \mathrm{O}$. Because of the way the field locking is performed, the magnetic field is the same for all measurements.

\section{RESULTS}

In order to derive the nuclear magnetic moment from the Larmor frequency in Eq. (1), the effective magnetic field $B$ needs to be known. Since the external field $B_{0}$ is modified by the bulk magnetic susceptibility of the host and by the NMR shielding of the nucleus in the host $\sigma, B$ can be, thus, expressed as [66]

$$
B=B_{0}[1+(1 / 3-\alpha) \kappa](1-\sigma),
$$

where $\kappa$ is the volume magnetic susceptibility and $\alpha$ is the shape factor (see the Appendix B for details). It is advantageous to use an approach where $\alpha \kappa$ or/and $\sigma$ cancel out, so we start by determining the ratio $R$ of the magnetic moment of ${ }^{26} \mathrm{Na}$ to that of ${ }^{23} \mathrm{Na}$ in the same ionic liquid host:

$$
R=\frac{\mu\left({ }^{26} \mathrm{Na}\right)}{\mu\left({ }^{23} \mathrm{Na}\right)}=\frac{\nu_{L}\left({ }^{26} \mathrm{Na}\right) I\left({ }^{26} \mathrm{Na}\right)}{\nu_{L}\left({ }^{23} \mathrm{Na}\right) I\left({ }^{23} \mathrm{Na}\right)} \frac{B\left({ }^{23} \mathrm{Na}\right)}{B\left({ }^{26} \mathrm{Na}\right)} .
$$

This value is independent of the NMR shielding (which is the same for ${ }^{26} \mathrm{Na}$ and ${ }^{23} \mathrm{Na}$ ) and includes only a correction due to the difference in bulk magnetic susceptibilities of our samples (Appendix B).
TABLE I. Larmor frequencies of ${ }^{26} \mathrm{Na}$ at $1.2 \mathrm{~T}$ and ${ }^{23} \mathrm{Na}$ at 7.05 T in BMIM-HCOO and EMIM-DCA, and the resulting ratio of the magnetic moments, $R$, based on Eq. (3). Errors in round brackets are due to the statistical uncertainties in the resonance frequencies. For $R$, this error includes only the uncertainty of the ${ }^{26} \mathrm{Na}$ resonance frequency, while the square brackets are due to other contributions, including the uncertainty of the ${ }^{23} \mathrm{Na}$ resonance frequency.

\begin{tabular}{lllc}
\hline \hline Liquid host & $\nu_{L}\left({ }^{26} \mathrm{Na}\right)(\mathrm{Hz})$ & $\nu_{L}\left({ }^{23} \mathrm{Na}\right)(\mathrm{Hz})$ & $R$ \\
\hline BMIM-HCOO & $8838826(14)$ & & \\
BMIM-HCOO & $8838834(12)$ & & $1.284956(2)[8]$ \\
EMIM-DCA & $8838838(10)$ & $79390300(100)$ & $1.284957(2)[8]$ \\
EMIM-DCA & $8838847(13)$ & & $1.284955(2)[8]$ \\
\hline \hline
\end{tabular}

The ${ }^{26} \mathrm{Na} \beta$-NMR spectra in EMIM-DCA and BMIMHCOO recorded at $1.2 \mathrm{~T}$ are shown in Fig. 3, while Table I shows the corresponding Larmor frequencies, together with reference frequencies for ${ }^{23} \mathrm{Na}$ at $7.05 \mathrm{~T}$. For each measurement, several spectra are analyzed, which differ in the observation time and in the coincident gate to determine the experimental $\beta$-decay asymmetry. The spectra are fitted with a flat baseline and Lorentzian profiles, which are expected due to a moderate rf power broadening. The data are also fitted using Gaussian profiles and a sloped baseline, with a negligible effect on the resonance frequency and its uncertainty. To extract the ${ }^{26} \mathrm{Na}$ resonance frequencies shown in Table I, spectra with a 250-ms observation time are used, as they provide the highest signal-to-noise ratio and the smallest nonstatistical scattering between data points. Because this scattering leads to the normalized (reduced) sum of residuals $\chi_{\text {red }}^{2}$ being higher than 1 , the fitted frequency uncertainty is scaled by $\sqrt{\chi_{\text {red }}^{2}}$, following the procedure by the Particle Data Group [67].

During the ${ }^{26} \mathrm{Na}$ measurements, the ${ }^{1} \mathrm{H}$ stabilizing NMR probe has a resonance frequency of $52008500(30) \mathrm{Hz}$. This frequency is $1050(150) \mathrm{Hz}$ lower than when the probe is placed at the sample position in the middle of the magnet, which leads to a corrected frequency of 52009550 (150) Hz. During the ${ }^{23} \mathrm{Na}$ measurements, the ${ }^{1} \mathrm{H}$ NMR Larmor frequency is $300131415(100) \mathrm{Hz}$.

Using the above Larmor frequencies and the magnetic susceptibility correction from Appendix B, the derived value of $R$ for each measurement is shown in Table I. The error in round brackets results from the statistical uncertainty on the ${ }^{26} \mathrm{Na}$ resonance position. The systematic error present in all measurements is shown in square brackets and includes systematic uncertainties in the resonance frequencies of ${ }^{1} \mathrm{H}$ and ${ }^{23} \mathrm{Na}$ and the uncertainty of the magnetic susceptibility correction. Here, the biggest contribution by far is the error in the frequency of ${ }^{1} \mathrm{H}$ during the $\beta$-NMR measurements, caused by the uncertainty in the position of the probe, which can be improved in the future. The final value of the ratio of $\mu\left({ }^{26} \mathrm{Na}\right)$ to $\mu\left({ }^{23} \mathrm{Na}\right)$ is 


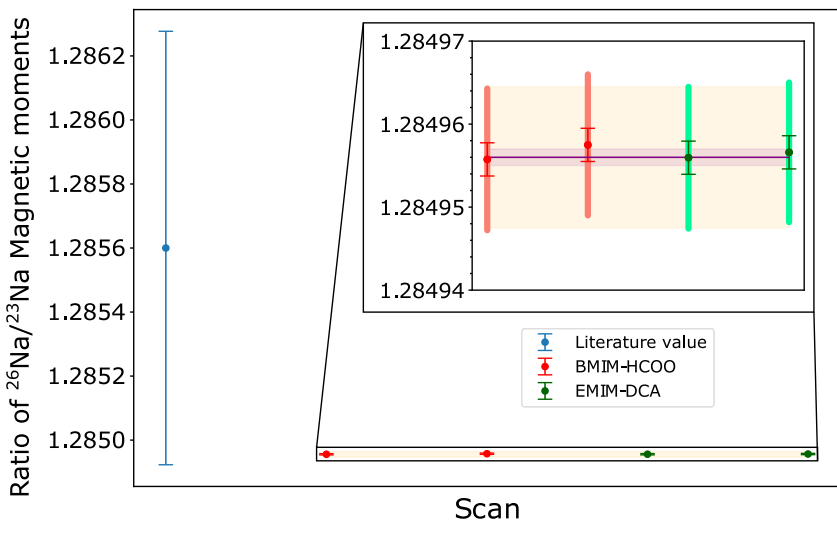

FIG. 4. Ratio of the magnetic moments of ${ }^{26} \mathrm{Na}$ and ${ }^{23} \mathrm{Na}$. Left: Literature value [68]; right: present study. Thin error-bar lines correspond to statistical uncertainty in the ${ }^{26} \mathrm{Na}$ Larmor frequency, and thick lines are the systematic uncertainties. The weighted average is represented by the purple line. The statistical uncertainty from all four measurements is indicated by the pink band, while the systematic uncertainty is shown by the broader orange band. For details, see the text.

$R=1.284956(1)[8]$ or $R=1.284956(8)$ with the uncertainties combined. Figure 4 shows the individual results in comparison to the literature value based on the hyperfinestructure measurement [68], which is 2 orders of magnitude less precise than our result. Our weighted average is indicated by the purple line. The purple shaded region represents the statistical uncertainty, while the orange region represents the systematic uncertainties.

In order to determine $\mu\left({ }^{26} \mathrm{Na}\right)$, a reliable reference $\mu\left({ }^{23} \mathrm{Na}\right)$ value is needed. In Nuclear Data Tables [17], the values of $\mu\left({ }^{26} \mathrm{Na}\right)$ based on atomic beam magnetic resonance (ABMR) and NMR experiments differ by $1.34 \times 10^{-4} \mu_{N}$, which is much larger than the individual error bars. This difference introduces an uncertainty that is larger than that of the frequency-ratio measurement in our $\beta$-NMR experiment. The above discrepancy stems from applying an obsolete diamagnetic correction [69] for the derivation of $\mu\left({ }^{23} \mathrm{Na}\right)$ from the experiments. This inconsistency can be corrected using $a b$ initio NMR shielding constants calculated for the species used in both experiments: a sodium atom in ABMR and an aqueous sodium ion in the NMR experiment. The technical details of NMR shielding calculations can be found in Appendix A.

The NMR shielding in the sodium atom calculated using the Dirac-Hartree-Fock (DHF) method is $637.1 \mathrm{ppm}$. The electron correlation contribution estimated using the DiracKohn-Sham (DKS) method with various density functional theory (DFT) functionals ranges from $0.06 \mathrm{ppm}$ for hybrid Perdew-Burke-Ernzerhof functional (PBE0) to $0.23 \mathrm{ppm}$ for hybrid 3-parameter Becke-Lee-Yang-Parr density functional (B3LYP). Coupled cluster codes for the NMR shielding of open-shell systems are not available. However, the accuracy of DKS correlation contributions can be estimated by the NMR shielding in the closed-shell $\mathrm{Na}^{+}$ ion and the difference between the electron correlation contributions in the sodium atom and sodium ion from the literature [70]. The nonrelativistic $\operatorname{CCSD}(\mathrm{T})$ correlation contribution calculated for the sodium ion, $-0.08 \mathrm{ppm}$, should not differ from the NMR shielding in the sodium

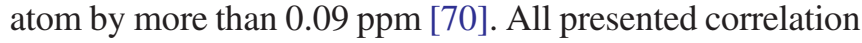
contributions suggest that the electron correlation effects for the NMR shielding in the sodium atom are small. Therefore, the NMR shielding in the sodium atom can be approximated with a very good accuracy by the DHF value. The electron correlation contributions can be used as an error estimate. Our final NMR shielding in the sodium atom, 637.1(2) ppm, is consistent with the shielding in Ref. [71]. The NMR shielding for the solvated sodium ion is approximated by the NMR shielding in a six-coordinated $\mathrm{Na}^{+}\left(\mathrm{H}_{2} \mathrm{O}\right)_{6}$ complex (the prevalent coordination number according the experiment [72]). The five-coordinated $\mathrm{Na}^{+}\left(\mathrm{H}_{2} \mathrm{O}\right)_{5}$ complex is used to estimate the error of the NMR shielding due to the structural uncertainty. The NMR shielding constants calculated using nonrelativistic and relativistic approximations for the $\mathrm{Na}^{+}\left(\mathrm{H}_{2} \mathrm{O}\right)_{6}$ complex are shown in Table II. The Hartree-Fock and DHF NMR shielding, electron correlation contributions $\left(\Delta_{\text {corr }}^{\mathrm{CCSD}}\right.$ and $\left.\Delta_{\text {corr }}^{\mathrm{CCSD}(\mathrm{T})}\right)$, and relativistic contributions $\left(\Delta_{\text {rel }}^{\mathrm{DHF}}, \Delta_{\text {rel }}^{\mathrm{DKS}}\right)$ reach good convergence with the basis size. The final NMR shielding for the $\mathrm{Na}^{+}\left(\mathrm{H}_{2} \mathrm{O}\right)_{6}$ complex, $582.0 \mathrm{ppm}$, is a composite value of (i) the nonrelativistic shielding calculated using the $\operatorname{CCSD}(\mathrm{T})$ method, (ii) relativistic correction $\left(\Delta_{\text {rel }}^{\mathrm{DKS}}\right)$, and (iii) the polarized continuum model (PCM) solvent contribution $\left(\Delta_{\text {solv }}^{\mathrm{PCM}}\right)$. All contributions entering the final

TABLE II. Sodium NMR shielding in the $\mathrm{Na}^{+}\left(\mathrm{H}_{2} \mathrm{O}\right)_{6}$ complex.

\begin{tabular}{lccc}
\hline \hline & $\mathrm{DZ}^{\mathrm{a}}$ & $\mathrm{TZ}^{\mathrm{a}}$ & $\mathrm{QZ}^{\mathrm{a}}$ \\
\hline $\mathrm{HF}$ & 578.588 & 578.814 & 579.150 \\
CCSD & 571.625 & 573.837 & 574.140 \\
CCSD(T) & 571.011 & 572.909 & 573.127 \\
$\Delta_{\text {corr }}^{\text {CCSD }}$ & -6.963 & -4.977 & -5.010 \\
$\Delta_{\text {corr }}^{\text {CCS }}$ & -0.614 & -0.928 & -1.013 \\
PBE0+PCM & 563.355 & 565.609 & 568.197 \\
PBE0 & 564.406 & 565.472 & 567.533 \\
$\Delta_{\text {solv }}^{\text {PCM }}$ & -1.051 & 0.137 & 0.664 \\
DHF $^{\text {DHF }}$ & 586.860 & 587.263 & 587.346 \\
$\Delta_{\text {rel }}^{\text {DHF }}$ & 578.980 & 579.089 & 579.151 \\
DKS/PBE0 & 7.880 & 8.174 & 8.195 \\
DKS/PBE0 $^{\text {b }}$ & 574.842 & 574.822 & 574.848 \\
$\Delta_{\text {rel }}^{\text {DKS }}$ & 567.007 & 566.694 & 566.688 \\
\hline \hline
\end{tabular}

${ }^{\mathrm{a}} \mathrm{DZ}, \mathrm{TZ}$, and QZ represent double- $\zeta$, triple- $\zeta$, and quadruple- $\zeta$ basis set respectively. cc-pCVXZ basis set series is used for sodium, and cc-pVXZ basis are used for hydrogen and oxygen. In relativistic calculations the basis sets are fully uncontracted.

${ }^{b}$ Nonrelativistic limit obtained with the speed of light rescaled by factor of 20 . 
NMR shielding are calculated using the quadruple- $\zeta$ (QZ) basis set.

The systematic error of the NMR shielding in the $\mathrm{Na}^{+}\left(\mathrm{H}_{2} \mathrm{O}\right)_{6}$ complex is evaluated as the square root of the sum of squares of the following errors. The structural uncertainty $(2 \mathrm{ppm})$ is evaluated as the difference between the CCSD NMR shielding for aqueous sodium complexes with the coordination number of five and six. The basis set incompleteness error (1 ppm) is estimated from the variations of the NMR shielding constants calculated using nonrelativistic HF method with Dunning and Jensen basis set series. The coupled cluster expansion truncation error is approximated by $\Delta_{\text {corr }}^{\mathrm{CCSD}(\mathrm{T})} \approx 1 \mathrm{ppm}$. Considering the convergence of the PCM solvent contribution $\left(\Delta_{\text {solv }}^{\mathrm{PCM}}\right)$, the error is estimated to be $1 \mathrm{ppm}$. The systematic error introduced by assuming an additivity of the electron correlation and the relativistic effects is negligible, as indicated by the small difference between the $\Delta_{\text {rel }}^{\mathrm{DHF}}$ and $\Delta_{\text {rel }}^{\mathrm{DKS}}$ relativistic corrections.

The final approximation of the NMR shielding of the aqueous sodium ion is $(582.0 \pm 2.6) \mathrm{ppm}$. This result is consistent with the NMR shielding in Ref. [73], but, in the present study, the error bar is reduced by a factor of 4 . This reduction is achieved by calculations with much larger basis sets, which lead to a better convergence of all contributions.

Table III presents the new values of the ${ }^{23} \mathrm{Na}$ reference magnetic moment rederived using our new NMR shielding constants. The ABMR-based magnetic moment is obtained using our $a b$ initio NMR shielding of the sodium atom and the original ABMR experiment [74]. The NMR-based magnetic moment is rederived using (i) our $a b$ initio NMR shielding of the aqueous sodium ion, (ii) the experimental frequency ratio 0.26451900 [75] of ${ }^{23} \mathrm{Na}$ in $0.1 \mathrm{M}$ $\mathrm{NaCl}$ water solution to the proton in tetramethylsilane (TMS), (iii) the reference proton magnetic moment $\mu\left({ }^{1} \mathrm{H}\right)=$ $2.792847348(7) \mu_{N}$ [76], and (iv) the reference NMR shielding of the proton in TMS $\sigma\left({ }^{1} \mathrm{H}\right)=33.480 \pm$ $0.5 \mathrm{pp} \mathrm{m}$ [77].

The newly extracted ABMR- and NMR-based values of the ${ }^{23} \mathrm{Na}$ nuclear magnetic dipole moment are now consistent within the error bars, and the discrepancy between them is decreased by a factor of approximately 30 .

TABLE III. $\mu\left({ }^{23} \mathrm{Na}\right) / \mu_{N}$ reference nuclear magnetic dipole moment from ABMR and NMR experiments.

\begin{tabular}{lcc}
\hline \hline & Old reference [17] & This work \\
\hline ABMR & $+2.217522(2)$ & $2.217495(2)^{\mathrm{a}}$ \\
NMR & $+2.2176556(6)$ & $2.217500(7)^{\mathrm{b}}$ \\
\hline \hline
\end{tabular}

${ }^{\mathrm{a}}$ Using the original ABMR experiment [74] and NMR shielding of the sodium atom $(637.1 \pm 0.2) \mathrm{ppm}$.

${ }^{b}$ Using the standard NMR frequency ratio of ${ }^{23} \mathrm{Na}$ in $\mathrm{NaCl}$ water solution to proton in TMS [75] and NMR shielding of $\mathrm{Na}^{+}\left(\mathrm{H}_{2} \mathrm{O}\right)_{6}(582.0 \pm 2.6) \mathrm{ppm}$. See the text for details on NMR shielding calculations.
TABLE IV. Magnetic moments of ${ }^{23,26-31} \mathrm{Na}$ determined in this work, compared to literature values $[42,68]$, and other nuclear properties relevant for NMR.

\begin{tabular}{lccccc}
\hline \hline Isotope & $I$ & $t_{1 / 2}(\mathrm{~ms})$ & $Q(\mathrm{mb})$ & Old $\mu\left(\mu_{N}\right)$ & New $\mu\left(\mu_{N}\right)$ \\
\hline${ }^{23} \mathrm{Na}$ & $3 / 2$ & Stable & $+106(1)$ & & $2.217500(7)^{\mathrm{a}}$ \\
${ }^{26} \mathrm{Na}$ & 3 & 1071 & $-5.3(2)$ & $2.851(2)$ & $2.849390(20)^{\mathrm{b}}$ \\
${ }^{27} \mathrm{Na}$ & $5 / 2$ & 301 & $-7.2(3)$ & $3.894(3)$ & $3.89212(24)$ \\
${ }^{28} \mathrm{Na}$ & 1 & 31 & $+39(1)$ & $2.420(2)$ & $2.41844(19)$ \\
${ }^{29} \mathrm{Na}$ & $3 / 2$ & 44 & $+86(3)$ & $2.457(2)$ & $2.45535(17)$ \\
${ }^{30} \mathrm{Na}$ & 2 & 48 & & $2.069(2)$ & $2.0681(11)$ \\
${ }^{31} \mathrm{Na}$ & $3 / 2$ & 17 & & $2.298(2)$ & $2.29670(17)$ \\
\hline \hline
\end{tabular}

${ }^{\mathrm{a}}$ Corrected $\mu\left({ }^{23} \mathrm{Na}\right)$ based on NMR experiment, Table III.

${ }^{\mathrm{b}}$ Based on our improved ratio of $\mu\left({ }^{26} \mathrm{Na}\right) / \mu\left({ }^{23} \mathrm{Na}\right)$.

For the derivation of the ${ }^{26} \mathrm{Na}$ nuclear magnetic dipole moment, the NMR-based ${ }^{23} \mathrm{Na}$ nuclear magnetic dipole moment is used, because the corresponding NMR shielding calculations for aqueous sodium complexes are based on a better approximation and the error bar is estimated more rigorously. The resulting ${ }^{26} \mathrm{Na}$ nuclear magnetic dipole moment is $2.849390(20) \mu_{N}$ (Table IV).

The new ${ }^{26} \mathrm{Na}$ nuclear magnetic dipole moment is consistent with the previous experimental value based on the hyperfine-structure measurement [68] within the error bar, but the present experiment and ab initio calculations improve its accuracy by 2 orders of magnitude, to $7 \mathrm{ppm}$. The largest contribution to this error bar comes from the uncertainty in the position of the ${ }^{1} \mathrm{H}$ NMR probe during the $\beta$-NMR experiment, which is 2 times larger than the uncertainty from NMR shielding and 3 times larger than the other experimental uncertainties. Experimental upgrades to provide a rigorous determination of the probe position could reduce the uncertainty of the ${ }^{26} \mathrm{Na}$ magnetic moment to the level of accuracy reached for the stable ${ }^{23} \mathrm{Na}$.

Magnetic moments which have been linked to ${ }^{26} \mathrm{Na}$ can also benefit from the improved accuracy of $\mu\left({ }^{26} \mathrm{Na}\right)$. This link is the case for ${ }^{27-31} \mathrm{Na}$, which are investigated using $\beta$-NMR in solid-state hosts at the collinear laser spectroscopy beam line at ISOLDE [42] and whose $g$ factors $g_{I}=$ $\mu /\left(I \mu_{N}\right)=\gamma \hbar / \mu_{N}$ are referenced to that of ${ }^{26} \mathrm{Na}$. Table IV presents our new values of the ${ }^{23} \mathrm{Na}$ and ${ }^{26} \mathrm{Na}$ magnetic moments, as well as the ${ }^{27-31} \mathrm{Na}$ magnetic moments obtained using our improved $\mu\left({ }^{26} \mathrm{Na}\right)$ and the aforementioned $g$ factors. Literature magnetic moments $[42,68]$ are also shown for comparison.

The new values of the ${ }^{27-29,31} \mathrm{Na}$ magnetic moments have a relative uncertainty of $70 \mathrm{ppm}$. This uncertainty is a tenfold improvement compared to the values deduced in Ref. [42] and up to 50 times more accurate than the values tabulated in the latest compilation of nuclear magnetic dipole and electric quadrupole moments [17] (for ${ }^{30} \mathrm{Na}$, it is, respectively, 2 [42] and 10 [17] times smaller). Previously, the uncertainty for ${ }^{27-31} \mathrm{Na}$ was dominated by the precision 
in the magnetic moment of the reference ${ }^{26} \mathrm{Na}$. At present, it is determined by the uncertainty in the ${ }^{27-31} \mathrm{Na} \beta$-NMR resonance frequency in solid-state hosts. If new measurements in liquid hosts are performed, this contribution could be decreased further to the ppm level.

\section{DISCUSSION AND FUTURE PERSPECTIVES}

To determine precise and accurate, shielding-corrected magnetic moments, two independent steps are needed. First, the Larmor frequency of the radioactive probe is measured relative to that of a stable NMR probe, e.g., ${ }^{1} \mathrm{H}$ or ${ }^{2} \mathrm{H}$ in water. This procedure removes the need for reference measurements relative to another radioactive probe nucleus, which is the current (time-consuming) reference method used in $\beta$-NMR. Furthermore, by using an ionic liquid as the host for the radioactive probe, a very precise Larmor frequency can be obtained, from which a precise (but still uncorrected) magnetic moment of a short-lived nucleus can be deduced relative to that of the stable $\left({ }^{1} \mathrm{H}\right.$ or $\left.{ }^{2} \mathrm{H}\right)$ probe. Second, the NMR shielding in the host is corrected, using one of two procedures. It can be calculated using modern calculation methods (if possible), or alternatively an independent NMR measurement has to be performed for the stable isotope of the element in the same host, again relative to the $\mathrm{H}$ reference. The latter approach is used here. The final accuracy on the magnetic moment then depends on the accuracy of the moment of the stable isotope, which can be deduced from former highprecision measurements in atoms, molecules, and liquids, in combination with state-of-the-art shielding calculations (as performed here).

The accurate magnetic moments of ${ }^{26-31} \mathrm{Na}$ presented above, together with that of ${ }^{23} \mathrm{Na}$, provide a set of NMR probes connected through the same NMR shielding. In this way, conventional NMR and the ultrasensitive $\beta$-NMR can be used to provide complementary information on chemical and biological processes, by probing different timescales and different nucleus-environment interactions (see Table IV). For example, with the very short-lived ${ }^{28} \mathrm{Na}$, one can probe processes with millisecond timescales, and with longer-lived ${ }^{26} \mathrm{Na}$ timescales of seconds, while stable ${ }^{23} \mathrm{Na}$ has a much longer observation window. Furthermore, quadrupole moments of ${ }^{26} \mathrm{Na}$ and ${ }^{27} \mathrm{Na}$ are, respectively, 20 and 15 times smaller compared to the stable ${ }^{23} \mathrm{Na}$. This difference results in a weaker interaction with the gradient of the electric field [78], leading to longer relaxation times and narrower resonances, which should permit the observation of NMR signals in hosts which display broad ${ }^{23} \mathrm{Na}$ resonances due to a strong quadrupolar interaction.

The approach presented here can be directly applied to other isotopic chains, thus expanding the palette of nuclei available for NMR spectroscopy. It can be combined with several techniques to polarize spins of short-lived nuclei. Some elements are easily polarized using element-specific laser optical pumping, as proven for several alkali and alkali-earth elements [2]. At the same time, universal polarization methods, such as pickup of polarized thermal neutrons, projectile fragmentation, or low-energy nuclear reactions, can be also used to produce polarized samples of radioactive isotopes; see Refs. $[1,79,80]$, and references therein.

Accurate magnetic moments of $\beta$-NMR probe nuclei are setting foundations for a novel referencing scheme in $\beta$-NMR spectroscopy. The method is based on measuring two Larmor frequencies simultaneously: for the radioactive probe in the chosen host material and a stable NMR probe like ${ }^{1} \mathrm{H}$ or ${ }^{2} \mathrm{H}$ in water placed in the experimental setup near the probe of interest. In this scheme, the absolute NMR shielding $\sigma_{X}$ instead of a chemical shift could be measured directly, following Eqs. (1) and (2):

$$
\sigma_{X}=1-\frac{\nu_{X}}{\nu_{Y}} \frac{\left|\mu_{Y}\right|}{\left|\mu_{X}\right|} \frac{I_{X}}{I_{Y}} \frac{1+\left(1 / 3-\alpha_{Y}\right) \kappa_{Y}}{1+\left(1 / 3-\alpha_{X}\right) \kappa_{X}}\left(1-\sigma_{Y}\right),
$$

where $X$ is the $\beta$-NMR probe nucleus and $Y$ is the reference conventional nucleus (e.g., ${ }^{1} \mathrm{H}$ in water). A description of the correction due to the difference in the bulk magnetic susceptibilities $\alpha \kappa$ is presented in Appendix B. Here, the $\beta$-NMR probe nucleus is related to the conventional NMR reference nucleus, which establishes a bridge between $\beta$-NMR spectroscopy and conventional NMR spectroscopy. This scheme offers the possibility to reference radioactive nuclei shielding to the stable nuclei not only within the isotopic chain, but also between different elements. This scheme removes the dependence of $\beta$-NMR spectroscopy on the ambiguous and often ad hoc standards defined for every element separately [29].

In this novel referencing scheme, the uncertainty of the NMR shielding $\sigma_{X}$ of $\beta$-NMR nuclei in different hosts, derived from Eq. (4), is defined primarily by the uncertainty in their magnetic moment. For ${ }^{26} \mathrm{Na}$, using the old value of the ${ }^{26} \mathrm{Na}$ magnetic moment leads to ${ }^{26} \mathrm{Na}$ NMR shielding values with a $\pm 700 \mathrm{ppm}$ error bar, which is about 10 times larger than the full range of chemical shifts for sodium [81]. In comparison, our new magnetic moment of ${ }^{26} \mathrm{Na}$ leads to 100 times more accurate shielding values $( \pm 7 \mathrm{ppm})$, which will be sufficient to distinguish between different sodium binding sites—see, e.g., Ref. [82] — and will enable comparisons to theoretical Na NMR shielding values [83-85].

All of the above innovations open the path for novel applications for $\beta$-NMR in chemistry and biology. One such application is the interaction of metal ions with biomolecules [86,87], which is important for the functions of living organisms (especially metal-ion-mediated folding of proteins [88] and nucleic acids [89]). For example, half of the proteins in our body contain metal ions, but their interactions and factors influencing them are still not fully understood. This lack is because many metal ions are silent for most spectroscopic techniques [87] and are very 
challenging for conventional NMR [71,90]. Yet, in NMR, metal nuclei are often very sensitive to small changes in geometry and coordination number, which gives rise to dozen-ppm shifts in resonance frequencies for many metals $[81,90]$. The application of $\beta$-NMR will allow this field to profit from up to a billion times increased sensitivity and access to readily available $\beta$-NMR probe nuclei with smaller or even no quadrupolar moment (see, e.g., Refs. $[3,42]$ ), giving rise to longer relaxation times and narrower resonances.

Using the advances presented here, pilot applications in biology are already planned. Among the biologically relevant metal ions, sodium and potassium play an important role in the formation and dynamics of special DNA structures, G-quadruplexes, which are promising targets for anticancer therapies [91]. Our present work prepares ${ }^{26} \mathrm{Na}$ to be an immediately applicable $\beta$-NMR probe to address this topic $[30,85,92,93]$. Presently, we are also exploring the most suitable potassium probes for G-quadruplex studies [30] and isotopes of several other elements relevant to protein folding [94].

In a very different field, namely, in nuclear structure, our research paves the way for addressing the open question about the distribution of neutrons inside atomic nuclei $[95,96]$. The neutron distribution impacts the properties of neutron stars [97], determines the limits of the nuclear landscape [98], and is responsible for novel phenomena and exotic structures in unstable nuclei [99]. It is especially important for light neutron-rich "halo" nuclei, consisting of a compact nuclear core and one or several loosely bound halo neutrons which are spatially extended [100,101]. As neutrons do not carry an electric charge, compared to protons their distribution is much more difficult to determine experimentally. However, because the neutron distribution is closely related to the distribution of nuclear magnetism, it can be addressed via the hyperfine anomaly, by combining the accurate magnetic moment with an accurate hyperfine structure measurement [102]. For example, in ${ }^{11} \mathrm{Be}$, the magnetism is mostly due to the halo neutron [55,56], so the hyperfine anomaly provides a direct probe of the halo structure [55,103]. Because the hyperfine structure of ${ }^{11} \mathrm{Be}$ is already known with high accuracy [56], the only missing experimental input to derive the neutron distribution from the hyperfine anomaly is an accurate value of the magnetic moment of ${ }^{11} \mathrm{Be}$, which can be achieved by applying the procedure presented in this work.

\section{CONCLUSIONS}

In summary, using ${ }^{26} \mathrm{Na}$ as an example, we have presented the first determination of a magnetic moment of a short-lived nucleus with ppm accuracy. This result represents an improvement by 2 orders of magnitude in comparison with a previous experiment and other $\beta$-NMR based measurements of magnetic moments. The procedure described in this article represents a general protocol for measurements of magnetic dipole moments of polarized $\beta$-decaying nuclei with high accuracy, reaching the accuracy for stable nuclei.

The innovations presented here bring the following advances for the ultrasensitive $\beta$-NMR technique: (i) elimination of the dependence of $\beta$-NMR spectroscopy on ambiguous and often ad hoc references. As a result, the uncertainty related to the $\beta$-NMR reference measurement can be removed from the analysis. In addition, the direct comparison of $\beta$-NMR and conventional NMR data bridges these two techniques. (ii) Saving scarce resources of radioactive beam for acquisition of more $\beta$-NMR data on the samples of interest, since a reference measurement on a $\beta$-NMR probe is not required. This solution will accelerate the application of $\beta$-NMR spectroscopy as an analytical tool. (iii) Link to ab initio predictions through the direct measurement of NMR shielding for $\beta$-NMR probes. This approach will facilitate the interpretation of $\beta$-NMR experiments.

These novel features have the potential to transform $\beta$-NMR spectroscopy into a more widely applicable technique, based on a palette of ultrasensitive $\beta$-NMR probes with accurate magnetic moments, allowing one to address problems that range from neutron distribution in exotic nuclei to interactions of metal ions with biomolecules.

\section{ACKNOWLEDGMENTS}

This work was supported by the European Research Council (Starting Grant No. 640465), CERN (BetDropNMR and gammaMRI MA Fund), the United Kingdom Science and Technology Facilities Council (No. ST/P004423/1), FWO-Vlaanderen in Belgium (No. G0B3415N), KU Leuven (No. GOA 15/010), EU project ENSAR2 (No. 654002), Slovak Research and Development Agency Grant (No. APVV-15-0105), European Regional Development Fund, Research and Innovation Operational Program (No. ITMS2014+: 313011W085), Polish National Science Centre (OPUS research Grant No. 2017/27/B/ST4/00485), the Ministry of Education, Youth and Sports of the Czech Republic (No. LM2015058), the Wolfgang Gentner Program of the German Federal Ministry of Education and Research (No. 05E15CHA), and the Swiss Excellence Scholarship program. Computational resources of the Slovak Academy of Sciences and the Slovak University of Technology were used (Projects No. ITMS 26230120002 and No. ITMS 26210120002). We thank the assistance of the ISOLDE technical team and that of L. Hemmingsen from Copenhagen University, M. Walczak from Poznań University of Technology, K. Szutkowski from A. Mickiewicz University in Poznań, M. Jankowski, R. Engel, and W. Neu from Oldenburg University, H. Heylen, A. Beaumont, and M. Van Stenis from CERN, V. Araujo from KU Leuven, A. Zhuravlova from Kiev 
University, K. Jackowski, M. Piersa, and E. Adamska from Warsaw University, J. Klimo, R. Urban, S. Komorovsky, G. Kantay, and J. Krajnak from the Slovak Academy of Sciences, E. Sistare from Geneva University, and M. Jaszuński from the Polish Academy of Sciences.

\section{APPENDIX A: AB INITIO NMR SHIELDING CALCULATIONS}

NMR shielding in the sodium atom with the doublet electronic ground state is calculated using the DiracHartree-Fock (DHF) method applying the paramagnetic NMR theory for open-shell systems $[70,104,105]$. DyallVXZ [106] basis set series are used $(X=\mathrm{D}, \mathrm{T}, \mathrm{Q}$ represents double- $\zeta$, triple- $\zeta$, and quadruple- $\zeta$ basis sets, respectively).

According to a recent experiment [72], the coordination number of the aqueous $\mathrm{Na}^{+}$ion depends on the $\mathrm{NaCl}$ solution concentration and varies between 5 and 6 . Therefore, NMR shielding of the $\mathrm{Na}^{+}$ion in the aqueous solution is calculated for model $\mathrm{Na}^{+}\left(\mathrm{H}_{2} \mathrm{O}\right)_{5}$ and $\mathrm{Na}^{+}\left(\mathrm{H}_{2} \mathrm{O}\right)_{6}$ complexes. Their structures are optimized using DFT with the B3LYP density functional [107-109] and Def2-TZVP basis set [110]. The D3 dispersion correction [111] is applied. A distorted octahedral structure $\left(\mathrm{D}_{2 h}\right.$ symmetry) is obtained for the $\mathrm{Na}^{+}\left(\mathrm{H}_{2} \mathrm{O}\right)_{6}$ complex, with an average Na-O distance of $2.386 \AA$. For $\mathrm{Na}^{+}\left(\mathrm{H}_{2} \mathrm{O}\right)_{5}$, the corresponding structure is found to be a trigonal bipyramid $\left(\mathrm{C}_{2 v}\right.$ symmetry) with an average $\mathrm{Na}-\mathrm{O}$ distance of $2.368 \AA$. The average $\mathrm{Na}-\mathrm{O}$ distances for both structures are in good agreement with the experimental $\mathrm{Na}-\mathrm{O}$ distances obtained with two different experimental methods giving $2.384 \pm$ 0.003 and $2.37 \pm 0.024 \AA$, respectively [72].

NMR shielding constants for aqueous sodium complexes are calculated using the nonrelativistic coupled cluster (CC) method with single and double excitations (CCSD) and with noniterative triple excitations $\operatorname{CCSD}(\mathrm{T})[112,113]$. All electrons are correlated. Dunning core-valence basis set series cc-pCVXZ [114] are used for sodium and valence series cc-pVXZ [115] for hydrogen and oxygen, combining basis sets with the same cardinal number $X(X=\mathrm{D}, \mathrm{T}, \mathrm{Q})$. In order to estimate the error due to incompleteness of the basis set, the pcS-n basis set series by Jensen [116] is also used. In all NMR shielding calculations, gauge-including atomic orbitals (GIAO) [117] are used.

The effect of the water solvent (outside the first solvation shell) on the NMR shielding in the sodium complex is incorporated by the PCM COSMO [118]. This effect is evaluated using DFT with the PBE0 functional $[119,120]$. The water dielectric constant of 78 is used in this implicit solvent model.

Relativistic corrections are calculated as the difference between the relativistic NMR shielding and the corresponding nonrelativistic limit using two different methods: the DKS method with the PBE0 functional and the DHF method. The nonrelativistic limit is obtained by rescaling the speed of light in the Hamiltonian by a factor of 20 . In the relativistic calculations, the Dunning basis sets are fully uncontracted, and a restricted magnetic balance scheme is employed to generate the small component basis set $[121,122]$. The nucleus is modeled by a Gaussian charge distribution [123].

For the structure optimization and for nonrelativistic DFT calculations of NMR shielding constants, the NWChem package is used [124]. Nonrelativistic coupled cluster NMR shielding calculations are carried out in the CFOUR [125] package. For relativistic NMR shielding calculations, the ReSpect $[126,127]$ program is used.

\section{APPENDIX B: EFFECT OF BULK MAGNETIC SUSCEPTIBILITY}

When using NMR to determine accurate nuclear magnetic moments or absolute NMR shielding, one should consider the differences in bulk magnetic susceptibility between the samples [66] [see Eqs. (2) and (4)]. This effect depends on the volume magnetic susceptibility of the host material $\kappa$ and on the geometry of the sample, reflected in the shape factor $\alpha$. For the shapes used in our studies, $\alpha \approx 0$ for the ${ }^{23} \mathrm{Na}$ and ${ }^{1} \mathrm{H}$ samples in conventional NMR and for ${ }^{26} \mathrm{Na}$ in $\beta$-NMR at CERN (cylinders parallel to the magnetic field [66] and a disk perpendicular to the magnetic field [128], respectively), whereas $\alpha \approx 1 / 2$ for the ${ }^{1} \mathrm{H}$ probe used at CERN (cylinder perpendicular to the field [66]).

In Eq. (3) for the ratio $R$ of the magnetic moments, the magnetic susceptibility corrections for ${ }^{23} \mathrm{Na}$ and ${ }^{26} \mathrm{Na}$ cancel out in the term $\nu_{L}\left({ }^{26} \mathrm{Na}\right) / \nu_{L}\left({ }^{23} \mathrm{Na}\right)$, due to the same $\alpha$ and $\kappa$. At the same time, $B\left({ }^{23} \mathrm{Na}\right) / B\left({ }^{26} \mathrm{Na}\right)=\nu_{L}\left({ }^{1} \mathrm{H}\right) / \nu_{L}^{\prime}\left({ }^{1} \mathrm{H}\right) \times$ $(1+\Delta)$, with $\Delta=\left(1-\frac{1}{6} \kappa_{\mathrm{H}_{2} \mathrm{O}}\right) /\left(1+\frac{1}{3} \kappa_{\mathrm{H}_{2} \mathrm{O}}\right)-1$ and a prime denoting the measurement at CERN. Using $\kappa_{\mathrm{H}_{2} \mathrm{O}}=$ $-9.04 \times 10^{-6}, \Delta \approx+4.5 \pm 0.5 \mathrm{ppm}$, where we assume a $10 \%$ uncertainty in the shape factors due to the finite size of the samples.

When using Eq. (4) to measure NMR shieldings with $\beta$-NMR, one must consider the shape factor $\alpha$ and the volume susceptibility $\kappa$ for the host of the reference nucleus (water in our case) and that of the $\beta$-NMR nucleus.

[1] G. Neyens, Nuclear Magnetic and Quadrupole Moments for Nuclear Structure Research on Exotic Nuclei, Rep. Prog. Phys. 66, 633 (2003).

[2] R. Neugart and G. Neyens, Nuclear Moments, in The Euroschool Lectures on Physics with Exotic Beams, Vol. II, edited by J. Al-Khalili and E. Roeckl (Springer, Berlin, 2006), pp. 135-189, https://doi.org/10.1007/3-540-337873 .4.

[3] G. Neyens, M. Kowalska, D. Yordanov, K. Blaum, P. Himpe, P. Lievens, S. Mallion, R. Neugart, N. Vermeulen, Y. Utsuno, and T. Otsuka, Measurement of the Spin and Magnetic Moment of ${ }^{31} \mathrm{Mg}$ : Evidence for a Strongly 
Deformed Intruder Ground State, Phys. Rev. Lett. 94, 022501 (2005).

[4] K. Asahi and K. Matsuta, Nuclear Electromagnetic Moments. $\beta$-NMR and Its Applications, Nucl. Phys. A693, 63 (2001).

[5] T. Ohtsubo, N. J. Stone, J. R. Stone, I. S. Towner, C. R. Bingham, C. Gaulard, U. Köster, S. Muto, J. Nikolov, K. Nishimura, G. S. Simpson, G. Soti, M. Veskovic, W. B. Walters, and F. Wauters, Magnetic Dipole Moment of the Doubly-Closed-Shell plus One Proton Nucleus ${ }^{49}$ Sc, Phys. Rev. Lett. 109, 032504 (2012).

[6] X. F. Yang et al., Isomer Shift and Magnetic Moment of the Long-Lived $1 / 2^{+}$Isomer in ${ }_{30}^{79} \mathrm{Zn}_{49}$ : Signature of Shape Coexistence near ${ }^{78} \mathrm{Ni}$, Phys. Rev. Lett. 116, 182502 (2016).

[7] J. Thielking, M. V. Okhapkin, P. Głowacki, D. M. Meier, L. Von Der Wense, B. Seiferle, C. E. Düllmann, P. G. Thirolf, and E. Peik, Laser Spectroscopic Characterization of the Nuclear-Clock Isomer ${ }^{229 m}$ Th, Nature (London) 556 , 321 (2018).

[8] N. Minkov and A. Pálffy, Theoretical Predictions for the Magnetic Dipole Moment of ${ }^{229 m}$ Th, Phys. Rev. Lett. 122, 162502 (2019).

[9] Y. Ichikawa et al., Interplay between Nuclear Shell Evolution and Shape Deformation Revealed by the Magnetic Moment of ${ }^{75} \mathrm{Cu}$, Nat. Phys. 15, 321 (2019).

[10] S. Raeder et al., Probing Sizes and Shapes of Nobelium Isotopes by Laser Spectroscopy, Phys. Rev. Lett. 120, 232503 (2018).

[11] G. A. Webb, Modern Magnetic Resonance, second ed. (Springer International, New York, 2018), pp. 1-2293.

[12] A. Naito, Experimental Approaches of NMR Spectroscopy: Methodology and Application to Life Science and Materials Science, 1st ed. (Springer, Singapore, 2018), pp. 1-636.

[13] E. M. Purcell, Research in Nuclear Magnetism, Science 118, 431 (1953).

[14] E. B. Baker and L. W. Build, NMR Spectrometer for All Nuclei Using a Frequency Synthesizer, Rev. Sci. Instrum. 34, 238 (1963).

[15] B. W. Epperlein, H. Kruger, and L. A. Schwenk, The Magnetic Moment of ${ }^{67} \mathrm{Zn}$ and the Shielding of Zinc Ions by Water, Phys. Lett. 45, 255 (1973).

[16] O. Lutz and H. Oehler, ${ }^{63} \mathrm{Cu}$ and ${ }^{65} \mathrm{Cu}$ Fourier Transform Nuclear Magnetic Resonance Studies, Z. Phys. A 21, 17 (1978).

[17] N. J. Stone, Table of Nuclear Magnetic Dipole and Electric Quadrupole Moments, At. Data Nucl. Data Tables 90, 75 (2005); N. J. Stone, Table of Recommended Nuclear Magnetic Dipole Moments: Part I, Long-Lived States, INDC (NDS) 079, 2019.

[18] T. Helgaker, M. Jaszuński, and K. Ruud, Ab Initio Methods for the Calculation of NMR Shielding and Indirect SpinSpin Coupling Constants, Chem. Rev. 99, 293 (1999).

[19] Calculation of NMR and EPR Parameters, edited by M. Kaupp, M. Bühl, and V. G. Malkin (Wiley, New York, 2004).

[20] A. Antušek, K. Jackowski, M. Jaszuński, W. Makulski, and M. Wilczek, Nuclear Magnetic Dipole Moments from NMR Spectra, Chem. Phys. Lett. 411, 111 (2005).

[21] M. Jaszuński, A. Antušek, P. Garbacz, K. Jackowski, W. Makulski, and M. Wilczek, The Determination of Accurate Nuclear Magnetic Dipole Moments and Direct Measure- ment of NMR Shielding Constants, Prog. Nucl. Magn. Reson. Spectrosc. 67, 49 (2012).

[22] B. Adrjan, W. Makulski, K. Jackowski, T. B. Demissie, K. Ruud, A. Antušek, and M. Jaszuński, NMR Absolute Shielding Scale and Nuclear Magnetic Dipole Moment of ${ }^{207} \mathrm{~Pb}$, Phys. Chem. Chem. Phys. 18, 16483 (2016).

[23] L. V. Skripnikov, S. Schmidt, J. Ullmann, C. Geppert, F. Kraus, B. Kresse, W. Nörtershäuser, A. F. Privalov, B. Scheibe, V. M. Shabaev, M. Vogel, and A. V. Volotka, New Nuclear Magnetic Moment of Resolving the Bismuth Hyperfine Puzzle, Phys. Rev. Lett. 120, 093001 (2018).

[24] A. Antušek, M. Repisky, M. Jaszuński, K. Jackowski, W. Makulski, and M. Misiak, Nuclear Magnetic Dipole Moment of ${ }^{209}$ Bi from NMR Experiments, Phys. Rev. A 98, 052509 (2018).

[25] A. Antušek and M. Repisky, NMR Absolute Shielding Scales and Nuclear Magnetic Dipole Moments of Transition Metal Nuclei, Phys. Chem. Chem. Phys. 22, 7065 (2020).

[26] M. G. H. Gustavsson and A.-M. Mårtensson-Pendrill, Need for Remeasurements of Nuclear Magnetic Dipole Moments, Phys. Rev. A 58, 3611 (1998).

[27] P. Indelicato, QED Tests with Highly Charged Ions, J. Phys. B 52, 232001 (2019).

[28] J. Ullmann et al., High Precision Hyperfine Measurements in Bismuth Challenge Bound-State Strong-Field QED, Nat. Commun. 8, 15484 (2017).

[29] K. Jackowski, M. Jaszuński, and M. Wilczek, Alternative Approach to the Standardization of NMR Spectra. Direct Measurement of Nuclear Magnetic Shielding in Molecules, J. Phys. Chem. A 114, 2471 (2010).

[30] B. Karg, M. Kowalska et al., Liquid $\beta$-NMR Studies of the Interaction of $\mathrm{Na}$ and $\mathrm{K}$ Cations with DNA G-Quadruplex Structures, INTC proposal, Report No. CERN-INTC2020-034/INTC-P-560, 2020, http://cds.cern.ch/record/ 2717964.

[31] N. Bloembergen and G. M. Temmer, Nuclear Magnetic Resonance of Aligned Radioactive Nuclei, Phys. Rev. 89, 883 (1953).

[32] D. A. Shirley, Radiative Detection of Nuclear Magnetic Resonance, Anal. Chem. 41, 69A (1969).

[33] A. Gottberg, M. Stachura, M. Kowalska, M. L. Bissell, V. Arcisauskaite, K. Blaum, A. Helmke, K. Johnston, K. Kreim, F. H. Larsen, R. Neugart, G. Neyens, R. F. G. Ruiz, D. Szunyogh, P. W. Thulstrup, D. T. Yordanov, and L. Hemmingsen, Billion-fold Enhancement in Sensitivity of Nuclear Magnetic Resonance Spectroscopy for Magnesium Ions in Solution, Chem. Phys. Chem. 15, 3929 (2014).

[34] D. Connor, Measurement of the Nuclear $g$ Factor of $\mathrm{Li}^{8}$, Phys. Rev. Lett. 3, 429 (1959).

[35] A. Winnacker, H. Ackermann, D. Dubbers, M. Grupp, P. Heitjans, and H.-J. Stockmann, Nuclear Moments of the $\beta$-Emitters ${ }^{108} \mathrm{Ag}$ and ${ }^{110} \mathrm{Ag}$, Nucl. Phys. A261, 261 (1976).

[36] E. Arnold, J. Bonn, R. Gegenwart, W. Neu, R. Neugart, E.-W. Otten, G. Ulm, and K. Wendt, Nuclear Spin and Magnetic Moment of ${ }^{11} \mathrm{Li}$, Phys. Lett. B 197, 311 (1987).

[37] H. Okuno, K. Asahi, H. Ueno, H. Izumi, H. Sato, M. Adachi, T. Nakamura, T. Kubo, N. Inabe, A. Yoshida, N. Fukunishi, T. Shimoda, H. Miyatake, N. Takahashi, 
W.-D. Schmidt-Ott, and M. Ishihara, Measurement of the Magnetic Moments of ${ }^{14} \mathrm{~B}$ and ${ }^{15} \mathrm{~B}$ Using Projectile Fragmentation Spin Polarization, Phys. Lett. B 354, 41 (1995).

[38] T. Onishi and K. Yoshida, Nuclear Magnetic Moment of Proton Drip-Line Nucleus ${ }^{9} \mathrm{C}$, Nucl. Phys. A588, 153 (1995).

[39] T. Minamisono, M. Tanigaki, Y. Nojiri, T. Onishi, T. Yamaguchi, M. Sasaki, T. Miyake, K. Minamisono, and K. Sato, Magnetic Moments of Proton Drip-Line Nuclei ${ }^{13} \mathrm{O}$ and ${ }^{9} \mathrm{C}$, Hyperfine Interact. 98, 519 (1996).

[40] H. Ueno et al., Magnetic Moments of ${ }^{17} \mathrm{~N}$ and ${ }^{17} \mathrm{~B}$, Phys. Rev. C 53, 2142 (1996).

[41] W. Geithner, S. Kappertz, M. Keim, P. Lievens, R. Neugart, L. Vermeeren, S. Wilbert, V. N. Fedoseyev, U. Koster, V. I. Mishin, V. Sebastian, and ISOLDE Collaboration, Measurement of the Magnetic Moment of the One-Neutron Halo Nucleus ${ }^{11}$ Be, Phys. Rev. Lett. 83, 3792 (1999).

[42] M. Keim, U. Georg, A. Klein, R. Neugart, M. Neuroth, S. Wilbert, P. Lievens, L. Vermeeren, B. A. Brown, and ISOLDE Collaboration, Measurement of the Electric Quadrupole Moments of ${ }^{26-29} \mathrm{Na}$, Eur. Phys. J. A 8, 31 (2000).

[43] H. Ogawa, K. Asahi, H. Ueno, H. Miyoshi, D. Kameda, N. Imai, K.-i. Yoneda, H. Watanabe, T. Suga, K.-i. Ohno, A. Yoshimi, W. Sato, K. Sakai, K. Yogo, Y. Kobayashi, A. Yoshida, T. Kubo, and M. Ishihara, Assignment of the Ground-State Spin-Parity for ${ }^{17} \mathrm{C}$ through g-Factor Measurement, Prog. Theor. Phys. Suppl. 146, 607 (2002).

[44] M. Mihara, R. Matsumiya, K. Shimomura, K. Matsuta, M. Fukuda, D. Ishikawa, J. Komurasaki, D. Nishimura, T. Nagasawa, T. Izumikawa, and T. Minamisono, Shallow Nitrogen Acceptor in $\mathrm{TiO}_{2}$ Studied by $\beta$-NMR Spectroscopy, Physica (Amsterdam) 401B-402B, 430 (2007).

[45] Z. Salman, O. Ofer, M. Radovic, H. Hao, M. B. Shalom, K. H. Chow, Y. Dagan, M. D. Hossain, C. D. P. Levy, W. A. MacFarlane, G. M. Morris, L. Patthey, M. R. Pearson, H. Saadaoui, T. Schmitt, D. Wang, and R. F. Kiefl, Nature of Weak Magnetism in $\mathrm{SrTiO}_{3} / \mathrm{LaAlO}_{3}$ Multilayers, Phys. Rev. Lett. 109, 257207 (2012).

[46] W. A. MacFarlane, C. B. L. Tschense, T. Buck, K. H. Chow, D. L. Cortie, A. N. Hariwal, R. F. Kiefl, D. Koumoulis, C. D. P. Levy, I. McKenzie, F. H. McGee, G. D. Morris, M. R. Pearson, Q. Song, D. Wang, Y. S. Hor, and R. J. Cava, $\beta$-Detected NMR of ${ }^{8} \mathrm{Li}^{+}$in $\mathrm{Bi}, \mathrm{Sb}$, and the Topological Insulator $\mathrm{Bi}_{0.9} \mathrm{Sb}_{0.1}$, Phys. Rev. $\mathrm{B} 90$, 214422 (2014).

[47] I. McKenzie, M. Harada, R. F. Kiefl, C. D. P. Levy, W. A. MacFarlane, G. D. Morris, S.-I. Ogata, M. R. Pearson, and J. Sugiyama, $\beta$-NMR Measurements of Lithium Ion Transport in Thin Films of Pure and Lithium-Salt-Doped Poly (Ethylene Oxide), J. Am. Chem. Soc. 136, 7833 (2014).

[48] D. L. Cortie, T. Buck, M. H. Dehn, R. F. Kiefl, C. D. P. Levy, R. M. L. McFadden, G. D. Morris, M. R. Pearson, Z. Salman, Y. Maeno, and W. A. MacFarlane, Spin Fluctuations in the Exotic Metallic State of $\mathrm{Sr}_{2} \mathrm{RuO}_{4}$ Studied with $\beta$-NMR, Phys. Rev. B 91, 241113(R) (2015).

[49] W. A. MacFarlane, Implanted-Ion $\beta$ NMR: A New Probe for Nanoscience, Solid-State NMR 68, 1 (2015).
[50] D. L. Cortie, T. Buck, M. H. Dehn, V. L. Karner, R. F. Kiefl, C. D. P. Levy, R. M. L. McFadden, G. D. Morris, I. McKenzie, M. R. Pearson, X. L. Wang, and W. A. MacFarlane, $\beta$-NMR Investigation of the Depth-Dependent Magnetic Properties of an Antiferromagnetic Surface, Phys. Rev. Lett. 116, 106103 (2016).

[51] J. Sugiyama, I. Umegaki, T. Uyama, R. M. L. McFadden, S. Shiraki, T. Hitosugi, Z. Salman, H. Saadaoui, G. D. Morris, W. A. MacFarlane, and R.F. Kiefl, Lithium Diffusion in Spinel $\mathrm{Li}_{4} \mathrm{Ti}_{5} \mathrm{O}_{12}$ and $\mathrm{LiTi}_{2} \mathrm{O}_{4}$ Films Detected with ${ }^{8} \mathrm{Li} \beta$-NMR, Phys. Rev. B 96, 094402 (2017).

[52] T. Sugihara et al., NMR Detection of Short-Lived $\beta$-Emitter ${ }^{12} \mathrm{~N}$ Implanted in Water, Hyperfine Interact. 238, 20 (2017).

[53] D. Szunyogh et al., Direct Observation of $\mathrm{Mg}^{2+}$ Complexes in Ionic Liquid Solutions by ${ }^{31} \mathrm{Mg} \beta-N M R$ Spectroscopy, Dalton Trans. 47, 14431 (2018).

[54] W. A. MacFarlane, T. J. Parolin, D. L. Cortie, K. H. Chow, M. D. Hossain, R. F. Kiefl, C. D. P. Levy, R. M. L. McFadden, G. D. Morris, M. R. Pearson, H. Saadaoui, Z. Salman, Q. Song, and D. Wang, ${ }^{8} \mathrm{Li}$ in the Cubic Insulator MgO, J. Phys. Conf. Ser. 551, 012033 (2014).

[55] M. Puchalski and K. Pachucki, Ground-State Hyperfine Splitting in the $\mathrm{Be}^{+}$Ion, Phys. Rev. A 89, 032510 (2014).

[56] A. Takamine, M. Wada, K. Okada, Y. Ito, P. Schury, F. Arai, I. Katayama, K. Imamura, Y. Ichikawa, H. Ueno, H. Wollnik, and H. Schuessler, Towards High Precision Measurements of Nuclear g-Factors for the Be Isotopes, Nucl. Instrum. Methods Phys. Res., Sect. B 376, 307 (2016).

[57] A. Bohr and V. F. Weisskopf, The Influence of Nuclear Structure on the Hyperfine Structure of Heavy Elements, Phys. Rev. 77, 94 (1950).

[58] F. F. Karpeshin and M. B. Trzhaskovskaya, The Theory of the Bohr Weisskopf Effect in the Hyperfine Structure, Nucl. Phys. A941, 66 (2015).

[59] J. R. Persson, Hyperfine Anomalies in $\mathrm{Gd}$ and Nd, Atoms 6, 2 (2018).

[60] M. J. Borge and K. Blaum, Focus on Exotic Beams at ISOLDE: A Laboratory Portrait, J. Phys. G 45, 010301 (2018).

[61] M. Kowalska, P. Aschenbrenner, M. Baranowski, M. L. Bissell, W. Gins, R. D. Harding, H. Heylen, G. Neyens, S. Pallada, N. Severijns, P. Velten, M. Walczak, F. Wienholtz, Z. Y. Xu, X.F. Yang, and D. Zakoucky, New Laser Polarization Line at the ISOLDE Facility, J. Phys. G 44, 084005 (2017).

[62] W. Gins, R. D. Harding, M. Baranowski, M. L. Bissell, R. F. G. Ruiz, M. Kowalska, G. Neyens, S. Pallada, N. Severijns, P. Velten, F. Wienholtz, Z. Y. Xu, X. F. Yang, and D. Zakoucky, A New Beamline for Laser SpinPolarization at ISOLDE, Nucl. Instrum. Methods Phys. Res., Sect. A 925, 24 (2019).

[63] H. H. Ginthard and J. J. Primas, Generating of Homogeneous Static Magnetic Fields, Patent No. 2953727, 1960, https://patentimages.storage.googleapis.com/66/ae/ 22/9e7cda1eed807b/US2953727.pdf.

[64] A. Abragam, The Principles of Nuclear Magnetism, International Series of Monographs on Physics (Clarendon, Oxford, 1989). 
[65] M. Bier and S. Dietrich, Vapour Pressure of Ionic Liquids, Mol. Phys. 108, 211 (2010).

[66] E. D. Becker, High Resolution NMR (Academic, New York, 2000).

[67] S. Eidelman et al. (Particle Data Group), Review of Particle Physics, Phys. Lett. B 592, 1 (2004).

[68] G. Huber, F. Touchard, S. Büttgenbach, C. Thibault, R. Klapisch, H. T. Duong, S. Liberman, J. Pinard, J. L. Vialle, P. Juncar, and P. Jacquinot, Spins, Magnetic Moments, and Isotope Shifts of ${ }^{21-31} \mathrm{Na}$ by High Resolution Laser Spectroscopy of the Atomic $D_{1}$ Line, Phys. Rev. C 18, 2342 (1978).

[69] P. Raghavan, Table of Nuclear Moments, At. Data Nucl. Data Tables 42, 189 (1989).

[70] N. C. Pyper, Do Theory and Experiment Agree for the Nuclear Shielding Difference between Alkali Atoms and Ions in Magnetic Resonance?, J. Phys. B 18, 1317 (1985).

[71] J. Mason, in Multinuclear NMR, edited by J. Mason (Springer, New York, 1987), pp. 1-660, https://doi.org/ 10.1007/978-1-4613-1783-8.

[72] M. Galib, M. D. Baer, L. B. Skinner, C. J. Mundy, T. Huthwelker, G. K. Schenter, C. J. Benmore, N. Govind, and J.L. Fulton, Revisiting the Hydration Structure of Aqueous $\mathrm{Na}^{+}$, J. Chem. Phys. 146, 084504 (2017).

[73] A. Antušek, D. Kędziera, A. Kaczmarek-Kędziera, and M. Jaszuński, Coupled Cluster Study of NMR Shielding of Alkali Metal Ions in Water Complexes and Magnetic Moments of Alkali Metal Nuclei, Chem. Phys. Lett. 532, 1 (2012).

[74] A. Beckmann, K. D. Böklen, and D. Elke, Precision Measurements of the Nuclear Magnetic Dipole Moments of ${ }^{6} \mathrm{Li},{ }^{7} \mathrm{Li},{ }^{23} \mathrm{Na},{ }^{39} \mathrm{~K}$ and ${ }^{41} \mathrm{~K}$, Z. Phys. 270 , 173 (1974).

[75] R. K. Harris, E. D. Becker, S. M. C. de Menezes, R. Goodfellow, and P. Granger, NMR Nomenclature. Nuclear Spin Properties and Conventions for Chemical Shifts (IUPAC Recommendations 2001), Pure Appl. Chem. 73, 1795 (2001); reprinted in Magn. Reson. Chem.40, 489 (2002).

[76] A. Mooser, S. Ulmer, K. Blaum, K. Franke, H. Kracke, C. Leiteritz, W. Quint, C. C. Rodegheri, C. Smorra, and J. Walz, Direct High-Precision Measurement of the Magnetic Moment of the Proton, Nature (London) 509, 596 (2014).

[77] P. Garbacz, K. Jackowski, W. Makulski, and R. E. Wasylishen, Nuclear Magnetic Shielding for Hydrogen in Selected Isolated Molecules, J. Phys. Chem. A 116, 11896 (2012).

[78] C. P. Slichter, Electric Quadrupole Effects, in Principles of Magnetic Resonance (Springer, Berlin, 1990), pp. 485502.

[79] H. Ackermann, P. Heitjans, and H. J. Stöckmann, Radiation Defects and Spin-Lattice Relaxation Studied with Neutron-Activated $\beta$ Emitters, Hyperfine Interact. 25, 395 (1985).

[80] B. Nojiri and Y. Deutch, Polarized $\beta$-Emitters for NMR Probes, Hyperfine Interact. 21, 197 (1985).

[81] T. P. Hanusa, Solution NMR of the Light Main Group Metals, Encycl. Inorg. Bioinorg. Chem., 1 (2015).
[82] M. Schmeisser, P. Illner, R. Puchta, A. Zahl, and R. van Eldik, Gutmann Donor and Acceptor Numbers for Ionic Liquids, Chem.-Eur. J. 18, 10969 (2012).

[83] J. A. Tossell, $\mathrm{Na}^{+}$Complexes with Crown Ethers: Calculation of ${ }^{23} \mathrm{Na}^{23} \mathrm{Na}$ NMR Shieldings and Quadrupole Coupling Constants, J. Phys. Chem. B 105, 11060 (2001).

[84] C. H. McMillen, C. K. Gren, T. P. Hanusa, and A. L. Rheingold, A Tetrameric Allyl Complex of Sodium, and Computational Modeling of the ${ }^{23} \mathrm{Na}$ Allyl Chemical Shift, Inorg. Chim. Acta 364, 61 (2010).

[85] A. Wong, F. W. Kotch, I. C. M. Kwan, J. T. Davis, and G. $\mathrm{Wu}$, Probing the Na+Binding Site in a Calix[4]Arene Guanosine Conjugate Dimer by Solid-State ${ }^{23} \mathrm{Na}$ NMR and Quantum Chemical Calculation, Chem. Commun. 2154 (2009).

[86] R. R. Crichton, Biological Inorganic Chemistry: A New Introduction to Molecular Structure and Function, third ed. (Elsevier, New York, 2019).

[87] R. R. Crichton and R. O. Louro, Practical Approaches to Biological Inorganic Chemistry, second ed. (Elsevier, New York, 2020).

[88] A. Sigel, Metal Ions in Biological Systems (CRC Press, Boca Raton, FL, 2001), pp. 1-744, https://doi.org/ 10.1201/9781482270150s.

[89] A. Sigel and H. Sigel, Interplay between Metal Ions and Nucleic Acids (Springer, Dordrecht, 2012), pp. 1-354, https://doi.org/10.1007/978-94-007-2172-2.

[90] F. G. Riddell, NMR Spectroscopy of Alkali Metal Nuclei in Solution, Encycl. Spectrosc. Spectrom. 2, 239 (2016).

[91] J. Carvalho, J. L. Mergny, G. F. Salgado, J. A. Queiroz, and C. Cruz, G-Quadruplex, Friend or Foe: The Role of the $G$-Quartet in Anticancer Strategies, Trends Mol. Med. 26, 848 (2020).

[92] G. Wu, A. Wong, Z. Gang, and J. T. Davis, Direct Detection of Potassium Cations Bound to G-Quadruplex Structures by Solid-State ${ }^{39} \mathrm{~K}$ NMR at 19.6 T, J. Am. Chem. Soc. 125, 7182 (2003).

[93] A. Wong, R. Ida, and G. Wu, Direct NMR Detection of the 'Invisible' Alkali Metal Cations Tightly Bound to GQuadruplex Structures, Biochem. Biophys. Res. Commun. 337, 363 (2005).

[94] A. Jancso, J. G. Correia, A. Gottberg, J. Schell, M. Stachura, D. Szunyogh, S. Pallada, D. C. Lupascu, M. Kowalska, and L. Hemmingsen, TDPAC and $\beta$-NMR Applications in Chemistry and Biochemistry, J. Phys. G 44, 064003 (2017).

[95] G. Hagen, A. Ekström, C. Forssén, G. R. Jansen, W. Nazarewicz, T. Papenbrock, K. A. Wendt, S. Bacca, N. Barnea, B. Carlsson, C. Drischler, K. Hebeler, M. Hjorth-Jensen, M. Miorelli, G. Orlandini, A. Schwenk, and J. Simonis, Neutron and Weak-Charge Distributions of the ${ }^{48} \mathrm{Ca}$ Nucleus, Nat. Phys. 12, 186 (2016).

[96] M. Thiel, C. Sfienti, J. Piekarewicz, C. J. Horowitz, and M. Vanderhaeghen, Neutron Skins of Atomic Nuclei: Per Aspera ad Astra, J. Phys. G 46, 093003 (2019).

[97] T. Aumann, C. A. Bertulani, F. Schindler, and S. Typel, Peeling Off Neutron Skins from Neutron-Rich Nuclei: Constraints on the Symmetry Energy from NeutronRemoval Cross Sections, Phys. Rev. Lett. 119, 262501 (2017). 
[98] J. Erler, N. Birge, M. Kortelainen, W. Nazarewicz, E. Olsen, A. M. Perhac, and M. Stoitsov, The Limits of the Nuclear Landscape, Nature (London) 486, 509 (2012).

[99] I. Tanihata, H. Hamagaki, O. Hashimoto, Y. Shida, N. Yoshikawa, K. Sugimoto, O. Yamakawa, T. Kobayashi, and N. Takahashi, Measurements of Interaction Cross Sections and Nuclear Radii in the Light p-Shell Region, Phys. Rev. Lett. 55, 2676 (1985).

[100] I. Tanihata, Neutron Halo Nuclei, J. Phys. G 22, 157 (1996).

[101] J. Al-Khalili, An Introduction to Halo Nuclei, in The Euroschool Lectures on Physics with Exotic Beams, Vol. I, edited by J. Al-Khalili and E. Roeckl (Springer, Berlin, 2004), pp. 77-112, https://doi.org/10.1007/978-3-54044490-9_3.

[102] H. H. Stroke, H. T. Duong, and J. Pinard, Bohr-Weisskopf Effect: Influence of the Distributed Nuclear Magnetization on HFS, Hyperfine Interact. 129, 319 (2000).

[103] Y.L. Parfenova and C. Leclercq-Willain, Hyperfine Anomaly in Be Isotopes and the Neutron Spatial Distribution: A Three-Cluster Model for ${ }^{9} \mathrm{Be}$, Phys. Rev. C 72, 054304 (2005).

[104] G. Malli and C. Froese, Nuclear Magnetic Shielding Constants Calculated from Numerical Hartree-Fock Wave Functions, Int. J. Quantum Chem. 1, 95 (2009).

[105] S. Komorovsky, M. Repisky, K. Ruud, O. L. Malkina, and V. G. Malkin, Four-Component Relativistic Density Functional Theory Calculations of NMR Shielding Tensors for Paramagnetic Systems, J. Phys. Chem. A 117, 14209 (2013).

[106] K. G. Dyall, Relativistic Quadruple-Zeta and Revised Triple-Zeta and Double-Zeta Basis Sets for the 4p, 5p, and $6 p$ Elements, Theor. Chem. Acc. 115, 441 (2006).

[107] A. D. Becke, Density-Functional Exchange-Energy Approximation with Correct Asymptotic Behavior, Phys. Rev. A 38, 3098 (1988).

[108] C. Lee, W. Yang, and R. G. Parr, Development of the ColleSalvetti Correlation-Energy Formula into a Functional of the Electron Density, Phys. Rev. B 37, 785 (1988).

[109] S. H. Vosko, L. Wilk, and M. Nusair, Accurate SpinDependent Electron Liquid Correlation Energies for Local Spin Density Calculations: A Critical Analysis, Can. J. Phys. 58, 1200 (1980).

[110] F. Weigend and R. Ahlrichs, Balanced Basis Sets of Split Valence, Triple Zeta Valence and Quadruple Zeta Valence Quality for H to Rn: Design and Assessment of Accuracy, Phys. Chem. Chem. Phys. 7, 3297 (2005).

[111] S. Grimme, S. Ehrlich, and L. Goerigk, Effect of the Damping Function in Dispersion Corrected Density Functional Theory, J. Comput. Chem. 32, 1456 (2011).

[112] J. Gauss and J. F. Stanton, Gauge-Invariant Calculation of Nuclear Magnetic Shielding Constants at the CoupledCluster Singles and Doubles Level, J. Chem. Phys. 102, 251 (1995).

[113] J. D. Watts, J. Gauss, and R. J. Bartlett, Coupled-Cluster Methods with Noniterative Triple Excitations for Restricted Open-Shell Hartree-Fock and Other General Single Determinant Reference Functions. Energies and Analytical Gradients, J. Chem. Phys. 98, 8718 (1993).
[114] K. L. Schuchardt, B. T. Didier, T. Elsethagen, L. Sun, V. Gurumoorthi, J. Chase, J. Li, and T. L. Windus, Basis Set Exchange: A Community Database for Computational Sciences, J. Chem. Inf. Model. 47, 1045 (2007).

[115] T. H. Dunning, Jr., Gaussian Basis Sets for Use in Correlated Molecular Calculations. I. The Atoms Boron through Neon and Hydrogen, J. Chem. Phys. 90, 1007 (1989).

[116] F. Jensen, Basis Set Convergence of Nuclear Magnetic Shielding Constants Calculated by Density Functional Methods, J. Chem. Theory Comput. 4, 719 (2008).

[117] K. Wolinski, J. F. Hinton, and P. Pulay, Efficient Implementation of the Gauge-Independent Atomic Orbital Method for NMR Chemical Shift Calculations, J. Am. Chem. Soc. 112, 8251 (1990).

[118] A. Klamt and G. Schuurmann, COSMO: A New Approach to Dielectric Screening in Solvents with Explicit Expressions for the Screening Energy and Its Gradient, J. Chem. Soc., Perkin Trans. 2, 799 (1993).

[119] J. P. Perdew, M. Ernzerhof, and K. Burke, Rationale for Mixing Exact Exchange with Density Functional Approximations, J. Chem. Phys. 105, 9982 (1996).

[120] C. Adamo and V. Barone, Toward Reliable Density Functional Methods without Adjustable Parameters: The PBEO Model, J. Chem. Phys. 110, 6158 (1999).

[121] S. Komorovský, M. Repiský, O. L. Malkina, V. G. Malkin, I. M. Ondík, and M. Kaupp, A Fully Relativistic Method for Calculation of Nuclear Magnetic Shielding Tensors with a Restricted Magnetically Balanced Basis in the Framework of the Matrix Dirac-Kohn-Sham Equation, J. Chem. Phys. 128, 104101 (2008).

[122] S. Komorovský, M. Repiský, O. L. Malkina, and V. G. Malkin, Fully Relativistic Calculations of NMR Shielding Tensors Using Restricted Magnetically Balanced Basis and Gauge Including Atomic Orbitals, J. Chem. Phys. 132, 154101 (2010).

[123] L. Visscher and K. G. Dyall, Dirac-Fock Atomic Electronic Structure Calculations Using Different Nuclear Charge Distributions, At. Data Nucl. Data Tables 67, 207 (1997).

[124] M. Valiev, E. J. Bylaska, N. Govind, K. Kowalski, T. P. Straatsma, H. J. J. van Dam, D. Wang, J. Nieplocha, E. Apra, T. L. Windus, and W. de Jong, NwChem: A Comprehensive and Scalable Open-Source Solution for Large Scale Molecular Simulations, Comput. Phys. Commun. 181, 1477 (2010).

[125] CFOUR, a quantum chemical program package written by J. F. Stanton, J. Gauss, M. E. Harding, P. G. Szalay with contributions from A. A. Auer, R. J. Bartlett, U. Benedikt, C. Berger, D. E. Bernholdt, J. Bomble, L. Cheng, O. Christiansen, M. Heckert, O. Heun, C. Huber, T.-C. Jagau, D. Jonsson, J. Jusélius, K. Klein, W. J. Lauderdale, D. A. Matthews, T. Metzroth, L. A. Mück, D. P. O'Neill, D. R. Price, E. Prochnow, C. Puzzarini, K. Ruud, F. Schiffmann, W. Schwalbach, C. Simmons, S. Stopkowicz, A. Tajti, J. Vázquez, F. Wang, J. D. Watts and the integral packages MOLECULE (J. Almlöf and P. R. Taylor), PROPS (P. R. Taylor), ABACUs (T. Helgaker, H. J. Aa. Jensen, P. Jørgensen, and J. Olsen), and ECP routines by A. V. Mitin 
and C. van Wüllen. For the current version, see http://www .cfour.de.

[126] ReSpect 5.0.1 (2018), relativistic spectroscopy DFT program of authors M. Repisky, S. Komorovsky, V. G. Malkin, O. L. Malkina, M. Kaupp, and K. Ruud, with contributions from R. Bast, R. Di Remigio, U. Ekstrom, M. Kadek, S. Knecht, L. Konecny, E. Malkin, and I. Malkin Ondik (see http://www.respectprogram.org).
[127] M. Repisky, S. Komorovsky, M. Kadek, L. Konecny, U. Ekström, E. Malkin, M. Kaupp, K. Ruud, O. L. Malkina, and V. G. Malkin, ReSpect: Relativistic spectroscopy DFT program package, J. Chem. Phys. 152, 184101 (2020).

[128] J. F. Schenck, The Role of Magnetic Susceptibility in Magnetic Resonance Imaging: MRI Magnetic Compatibility of the First and Second Kinds, Med. Phys. 23, 815 (1996). 